\title{
The form of solutions and periodic nature for some rational difference equations systems
}

\author{
M. M. El-Dessoky ${ }^{a, b, *}$, E. M. Elsayed ${ }^{a, b}$, E. O. Alzahranib
}

${ }^{a}$ King Abdulaziz University, Faculty of Science, Mathematics Department, P. O. Box 80203, Jeddah 21589, Saudi Arabia.

${ }^{b}$ Department of Mathematics, Faculty of Science, Mansoura University, Mansoura 35516, Egypt.

Communicated by N. Shahzad

\begin{abstract}
In this paper, we investigate the expressions of solutions and the periodic nature of the following systems of rational difference equations with order four

$$
x_{n+1}=\frac{y_{n-3}}{ \pm 1 \pm y_{n} z_{n-1} x_{n-2} y_{n-3}}, y_{n+1}=\frac{z_{n-3}}{ \pm 1 \pm z_{n} x_{n-1} y_{n-2} z_{n-3}}, z_{n+1}=\frac{x_{n-3}}{ \pm 1 \pm x_{n} y_{n-1} z_{n-2} x_{n-3}},
$$

with initial conditions $x_{-3}, x_{-2}, x_{-1}, x_{0}, y_{-3}, y_{-2}, y_{-1}, y_{0}, z_{-3}, z_{-2}, z_{-1}$ and $z_{0}$ which are arbitrary real numbers. (C)2016 All rights reserved.
\end{abstract}

Keywords: Difference equations, recursive sequences, stability, periodic solution, system of difference equations.

2010 MSC: 39A10.

\section{Introduction}

The goal of this paper is to obtain the form of the solutions and the periodicity character of some systems of rational difference equations

$$
x_{n+1}=\frac{y_{n-3}}{ \pm 1 \pm y_{n} z_{n-1} x_{n-2} y_{n-3}}, y_{n+1}=\frac{z_{n-3}}{ \pm 1 \pm z_{n} x_{n-1} y_{n-2} z_{n-3}}, z_{n+1}=\frac{x_{n-3}}{ \pm 1 \pm x_{n} y_{n-1} z_{n-2} x_{n-3}},
$$

with initial conditions, which are arbitrary real numbers.

\footnotetext{
${ }^{*}$ Corresponding author

Email addresses: dessokym@mans.edu.eg (M. M. El-Dessoky), emelsayed@mans.edu.eg (E. M. Elsayed), eoalzahrani@kau.edu.sa (E. O. Alzahrani)
} 
Difference equations appear naturally as discrete analogues and as numerical solutions of differential and delay differential equations having applications in biology, ecology, economy, physics, and so on. Although difference equations are very simple in form, it is extremely difficult to understand thoroughly the behaviors of their solution, see [1, 4, 6 6 , 11, 13, 15, 19, 20, 22, 24, 25, 27, 31, 32] and the references cited therein. Recently, a great effort has been made in studying the qualitative analysis of rational difference equations and rational difference equations system, see [4 31, 33, 34].

Din et al. [8] investigated the qualitative behavior of the following competitive system of rational difference equations

$$
x_{n+1}=\frac{\alpha_{1}+\beta_{1} x_{n-1}}{a_{1}+b_{1} y_{n}}, y_{n+1}=\frac{\alpha_{2}+\beta_{2} y_{n-1}}{a_{2}+b_{2} x_{n}} .
$$

Elsayed et al. [15] studied the form of the solutions and the periodicity of the following rational systems of rational difference equations

$$
x_{n+1}=\frac{x_{n-5}}{1-x_{n-5} y_{n-2}}, y_{n+1}=\frac{y_{n-5}}{ \pm 1 \pm y_{n-5} x_{n-2}} .
$$

Grove et al. [19] studied the existence and behavior of solution of the rational system

$$
x_{n+1}=\frac{a}{x_{n}}+\frac{b}{y_{n}}, y_{n+1}=\frac{c}{x_{n}}+\frac{d}{y_{n}} .
$$

The behavior of positive solutions of the following system

$$
x_{n+1}=\frac{x_{n-1}}{1+x_{n-1} y_{n}}, y_{n+1}=\frac{y_{n-1}}{1+y_{n-1} x_{n}},
$$

were studied by Kurbanli et al. in [24].

Özban [25] has investigated the positive solution of the system of rational difference equations

$$
x_{n+1}=\frac{a}{y_{n-3}}, y_{n+1}=\frac{b y_{n-3}}{x_{n-q} y_{n-q}} .
$$

Also, Touafek et al. [27] studied the periodicity and gave the form of the solutions of the following systems

$$
x_{n+1}=\frac{y_{n}}{x_{n-1}\left( \pm 1 \pm y_{n}\right)}, y_{n+1}=\frac{x_{n}}{y_{n-1}\left( \pm 1 \pm x_{n}\right)} .
$$

Elabbasy et al. [11] obtained the solution of particular cases of the following general system of difference equations

$$
x_{n+1}=\frac{a_{1}+a_{2} y_{n}}{a_{3} z_{n}+a_{4} x_{n-1} z_{n}}, y_{n+1}=\frac{b_{1} z_{n-1}+b_{2} z_{n}}{b_{3} x_{n} y_{n}+b_{4} x_{n} y_{n-1}}, z_{n+1}=\frac{c_{1} z_{n-1}+c_{2} z_{n}}{c_{3} x_{n-1} y_{n-1}+c_{4} x_{n-1} y_{n}+c_{5} x_{n} y_{n}} .
$$

2. On the systems: $x_{n+1}=\frac{y_{n-3}}{1 \pm y_{n} z_{n-1} x_{n-2} y_{n-3}}, \quad y_{n+1}=\frac{z_{n-3}}{1 \pm z_{n} x_{n-1} y_{n-2} z_{n-3}}, z_{n+1}=\frac{x_{n-3}}{1 \pm x_{n} y_{n-1} z_{n-2} x_{n-3}}$

In this section, we study the solutions of the system of three difference equations in the following form

$$
\begin{aligned}
x_{n+1} & =\frac{y_{n-3}}{1+y_{n} z_{n-1} x_{n-2} y_{n-3}}, y_{n+1}=\frac{z_{n-3}}{1+z_{n} x_{n-1} y_{n-2} z_{n-3}}, \\
z_{n+1} & =\frac{x_{n-3}}{1+x_{n} y_{n-1} z_{n-2} x_{n-3}}, \quad n=0,1, \ldots,
\end{aligned}
$$

with nonzero real initial conditions $x_{-3}, x_{-2}, x_{-1}, x_{0}, y_{-3}, y_{-2}, y_{-1}, y_{0}, z_{-3}, z_{-2}, z_{-1}, z_{0}$.

Theorem 2.1. Suppose that $\left\{x_{n}, y_{n}, z_{n}\right\}$ are solutions of system (2.1), then for $n=0,1,2, \ldots$, we see that

$$
\begin{aligned}
& x_{12 n-3}=a \prod_{i=0}^{n-1} \frac{(1+12 i a d g l)(1+(12 i+4) \text { adgl })(1+(12 i+8) \text { adgl })}{(1+(12 i+1) a d g l)(1+(12 i+5) a d g l)(1+(12 i+9) a d g l)}, \\
& x_{12 n-2}=b \prod_{i=0}^{n-1} \frac{(1+(12 i+1) \text { behm })(1+(12 i+5) \text { behm })(1+(12 i+9) b e h m)}{(1+(12 i+2) b e h m)(1+(12 i+6) b e h m)(1+(12 i+10) \text { behm })},
\end{aligned}
$$




$$
\begin{aligned}
& x_{12 n-1}=c \prod_{i=0}^{n-1} \frac{(1+(12 i+2) c f k o)(1+(12 i+6) c f k o)(1+(12 i+10) c f k o)}{(1+(12 i+3) c f k o)(1+(12 i+7) c f k o)(1+(12 i+11) c f k o)}, \\
& x_{12 n}=d \prod_{i=0}^{n-1} \frac{(1+(12 i+3) a d g l)(1+(12 i+7) a d g l)(1+(12 i+11) a d g l)}{(1+(12 i+4) a d g l)(1+(12 i+8) a d g l)(1+(12 i+12) a d g l)}, \\
& x_{12 n+1}=\frac{e}{(1+\text { behm })} \prod_{i=0}^{n-1} \frac{(1+(12 i+4) \text { behm })(1+(12 i+8) \text { behm })(1+(12 i+12) \text { behm })}{(1+(12 i+5) \text { behm })(1+(12 i+9) \text { behm })(1+(12 i+13) \text { behm })}, \\
& x_{12 n+2}=\frac{f(1+c f k o)}{(1+2 c f k o)} \prod_{i=0}^{n-1} \frac{(1+(12 i+5) c f k o)(1+(12 i+9) c f k o)(1+(12 i+13) c f k o)}{(1+(12 i+6) c f k o)(1+(12 i+10) c f k o)(1+(12 i+14) c f k o)}, \\
& x_{12 n+3}=\frac{g(1+2 a d g l)}{(1+3 a d g l)} \prod_{i=0}^{n-1} \frac{(1+(12 i+6) a d g l)(1+(12 i+10) a d g l)(1+(12 i+14) a d g l)}{(1+(12 i+7) a d g l)(1+(12 i+11) a d g l)(1+(12 i+15) a d g l)}, \\
& x_{12 n+4}=\frac{h(1+3 b e h m)}{(1+4 b e h m)} \prod_{i=0}^{n-1} \frac{(1+(12 i+7) b e h m)(1+(12 i+11) b e h m)(1+(12 i+15) \text { behm })}{(1+(12 i+8) b e h m)(1+(12 i+12) b e h m)(1+(12 i+16) b e h m)}, \\
& x_{12 n+5}=\frac{k(1+4 c f k o)}{(1+c f k o)(1+5 c f k o)} \prod_{i=0}^{n-1} \frac{(1+(12 i+8) c f k o)(1+(12 i+12) c f k o)(1+(12 i+16) c f k o)}{(1+(12 i+9) c f k o)(1+(12 i+13) c f k o)(1+(12 i+17) c f k o)}, \\
& x_{12 n+6}=\frac{l(1+a d g l)(1+5 a d g l)}{(1+2 a d g l)(1+6 a d g l)} \prod_{i=0}^{n-1} \frac{(1+(12 i+9) a d g l)(1+(12 i+13) a d g l)(1+(12 i+17) a d g l)}{(1+(12 i+10) a d g l)(1+(12 i+14) a d g l)(1+(12 i+18) a d g l)}, \\
& x_{12 n+7}=\frac{m(1+2 b e h m)(1+6 b e h m)}{(1+3 b e h m)(1+7 b e h m)} \prod_{i=0}^{n-1} \frac{(1+(12 i+10) \text { behm })(1+(12 i+14) \text { behm })(1+(12 i+18) \text { behm })}{(1+(12 i+11) \text { behm })(1+(12 i+15) \text { behm })(1+(12 i+19) \text { behm })} \text {, } \\
& x_{12 n+8}=\frac{o(1+3 c f k o)(1+7 c f k o)}{(1+4 c f k o)(1+8 c f k o)} \prod_{i=0}^{n-1} \frac{(1+(12 i+11) c f k o)(1+(12 i+15) c f k o)(1+(12 i+19) c f k o)}{(1+(12 i+12) c f k o)(1+(12 i+16) c f k o)(1+(12 i+20) c f k o)}, \\
& y_{12 n-3}=e \prod_{i=0}^{n-1} \frac{(1+(12 i) b e h m)(1+(12 i+4) b e h m)(1+(12 i+8) b e h m)}{(1+(12 i+1) \text { behm })(1+(12 i+5) \text { behm })(1+(12 i+9) b e h m)}, \\
& y_{12 n-2}=f \prod_{i=0}^{n-1} \frac{(1+(12 i+1) c f k o)(1+(12 i+5) c f k o)(1+(12 i+9) c f k o)}{(1+(12 i+2) c f k o)(1+(12 i+6) c f k o)(1+(12 i+10) c f k o)}, \\
& y_{12 n-1}=g \prod_{i=0}^{n-1} \frac{(1+(12 i+2) a d g l)(1+(12 i+6) a d g l)(1+(12 i+10) a d g l)}{(1+(12 i+3) a d g l)(1+(12 i+7) a d g l)(1+(12 i+11) a d g l)}, \\
& y_{12 n}=h \prod_{i=0}^{n-1} \frac{(1+(12 i+3) \text { behm })(1+(12 i+7) \text { behm })(1+(12 i+11) \text { behm })}{(1+(12 i+4) \text { behm })(1+(12 i+8) \text { behm })(1+(12 i+12) \text { behm })}, \\
& y_{12 n+1}=\frac{k}{(1+c f k o)} \prod_{i=0}^{n-1} \frac{(1+(12 i+4) c f k o)(1+(12 i+8) c f k o)(1+(12 i+12) c f k o)}{(1+(12 i+5) c f k o)(1+(12 i+9) c f k o)(1+(12 i+13) c f k o)} \text {, } \\
& y_{12 n+2}=\frac{l(1+a d g l)}{(1+2 a d g l)} \prod_{i=0}^{n-1} \frac{(1+(12 i+5) a d g l)(1+(12 i+9) a d g l)(1+(12 i+13) a d g l)}{(1+(12 i+6) a d g l)(1+(12 i+10) a d g l)(1+(12 i+14) a d g l)}, \\
& y_{12 n+3}=\frac{m(1+2 b e h m)}{(1+3 b e h m)} \prod_{i=0}^{n-1} \frac{(1+(12 i+6) \text { behm })(1+(12 i+10) \text { behm })(1+(12 i+14) \text { behm })}{(1+(12 i+7) \text { behm })(1+(12 i+11) b e h m)(1+(12 i+15) b e h m)}, \\
& y_{12 n+4}=\frac{o(1+3 c f k o)}{(1+4 c f k o)} \prod_{i=0}^{n-1} \frac{(1+(12 i+7) c f k o)(1+(12 i+11) c f k o)(1+(12 i+15) c f k o)}{(1+(12 i+8) c f k o)(1+(12 i+12) c f k o)(1+(12 i+16) c f k o)}, \\
& y_{12 n+5}=\frac{a(1+4 a d g l)}{(1+a d g l)(1+5 a d g l)} \prod_{i=0}^{n-1} \frac{(1+(12 i+8) a d g l)(1+(12 i+12) a d g l)(1+(12 i+16) a d g l)}{(1+(12 i+9) a d g l)(1+(12 i+13) a d g l)(1+(12 i+17) a d g l)}, \\
& y_{12 n+6}=\frac{b(1+\text { behm })(1+5 b e h m)}{(1+2 b e h m)(1+6 b e h m)} \prod_{i=0}^{n-1} \frac{(1+(12 i+9) \text { behm })(1+(12 i+13) \text { behm })(1+(12 i+17) \text { behm })}{(1+(12 i+10) \text { behm })(1+(12 i+14) \text { behm })(1+(12 i+18) \text { behm })} \text {, } \\
& y_{12 n+7}=\frac{c(1+2 c f k o)(1+6 c f k o)}{(1+3 c f k o)(1+7 c f k o)} \prod_{i=0}^{n-1} \frac{(1+(12 i+10) c f k o)(1+(12 i+14) c f k o)(1+(12 i+18) c f k o)}{(1+(12 i+11) c f k o)(1+(12 i+15) c f k o)(1+(12 i+19) c f k o)}
\end{aligned}
$$




$$
y_{12 n+8}=\frac{d(1+3 a d g l)(1+7 a d g l)}{(1+4 a d g l)(1+8 a d g l)} \prod_{i=0}^{n-1} \frac{(1+(12 i+11) a d g l)(1+(12 i+15) a d g l)(1+(12 i+19) a d g l)}{(1+(12 i+12) a d g l)(1+(12 i+16) a d g l)(1+(12 i+20) a d g l)}
$$

and

$$
\begin{aligned}
& z_{12 n-3}=k \prod_{i=0}^{n-1} \frac{(1+(12 i) c f k o)(1+(12 i+4) c f k o)(1+(12 i+8) c f k o)}{(1+(12 i+1) c f k o)(1+(12 i+5) c f k o)(1+(12 i+9) c f k o)}, \\
& z_{12 n-2}=l \prod_{i=0}^{n-1} \frac{(1+(12 i+1) a d g l)(1+(12 i+5) a d g l)(1+(12 i+9) a d g l)}{(1+(12 i+2) a d g l)(1+(12 i+6) a d g l)(1+(12 i+10) a d g l)}, \\
& z_{12 n-1}=m \prod_{i=0}^{n-1} \frac{(1+(12 i+2) \text { behm })(1+(12 i+6) \text { behm })(1+(12 i+10) \text { behm })}{(1+(12 i+3) \text { behm })(1+(12 i+7) \text { behm })(1+(12 i+11) \text { behm })}, \\
& z_{12 n}=o \prod_{i=0}^{n-1} \frac{(1+(12 i+3) c f k o)(1+(12 i+7) c f k o)(1+(12 i+11) c f k o)}{(1+(12 i+4) c f k o)(1+(12 i+8) c f k o)(1+(12 i+12) c f k o)}, \\
& z_{12 n+1}=\frac{a}{(1+a d g l)} \prod_{i=0}^{n-1} \frac{(1+(12 i+4) a d g l)(1+(12 i+8) a d g l)(1+(12 i+12) a d g l)}{(1+(12 i+5) a d g l)(1+(12 i+9) a d g l)(1+(12 i+13) a d g l)}, \\
& z_{12 n+2}=\frac{b(1+\text { behm })}{(1+2 b e h m)} \prod_{i=0}^{n-1} \frac{(1+(12 i+5) \text { behm })(1+(12 i+9) \text { behm })(1+(12 i+13) \text { behm })}{(1+(12 i+6) \text { behm })(1+(12 i+10) \text { behm })(1+(12 i+14) \text { behm })}, \\
& z_{12 n+3}=\frac{c(1+2 c f k o)}{(1+3 c f k o)} \prod_{i=0}^{n-1} \frac{(1+(12 i+6) c f k o)(1+(12 i+10) c f k o)(1+(12 i+14) c f k o)}{(1+(12 i+7) c f k o)(1+(12 i+11) c f k o)(1+(12 i+15) c f k o)}, \\
& z_{12 n+4}=\frac{d(1+3 a d g l)}{(1+4 a d g l)} \prod_{i=0}^{n-1} \frac{(1+(12 i+7) a d g l)(1+(12 i+11) a d g l)(1+(12 i+15) a d g l)}{(1+(12 i+8) a d g l)(1+(12 i+12) a d g l)(1+(12 i+16) a d g l)}, \\
& z_{12 n+5}=\frac{e(1+4 b e h m)}{(1+\text { behm })(1+5 b e h m)} \prod_{i=0}^{n-1} \frac{(1+(12 i+8) \text { behm })(1+(12 i+12) \text { behm })(1+(12 i+16) \text { behm })}{(1+(12 i+9) \text { behm })(1+(12 i+13) \text { behm })(1+(12 i+17) \text { behm })}, \\
& z_{12 n+6}=\frac{f(1+c f k o)(1+5 c f k o)}{(1+2 c f k o)(1+6 c f k o)} \prod_{i=0}^{n-1} \frac{(1+(12 i+9) c f k o)(1+(12 i+13) c f k o)(1+(12 i+17) c f k o)}{(1+(12 i+10) c f k o)(1+(12 i+14) c f k o)(1+(12 i+18) c f k o)}, \\
& z_{12 n+7}=\frac{g(1+2 a d g l)(1+6 a d g l)}{(1+3 a d g l)(1+7 a d g l)} \prod_{i=0}^{n-1} \frac{(1+(12 i+10) a d g l)(1+(12 i+14) a d g l)(1+(12 i+18) a d g l)}{(1+(12 i+11) a d g l)(1+(12 i+15) a d g l)(1+(12 i+19) a d g l)}, \\
& z_{12 n+8}=\frac{h(1+3 b e h m)(1+7 b e h m)}{(1+4 b e h m)(1+8 b e h m)} \prod_{i=0}^{n-1} \frac{(1+(12 i+11) \text { behm })(1+(12 i+15) \text { behm })(1+(12 i+19) \text { behm })}{(1+(12 i+12) \text { behm })(1+(12 i+16) \text { behm })(1+(12 i+20) \text { behm })},
\end{aligned}
$$

where $x_{-3}=a, x_{-2}=b, x_{-1}=c, x_{0}=d, y_{-3}=e, y_{-2}=f, y_{-1}=g, y_{0}=h, z_{-3}=k, z_{-2}=l, z_{-1}=$ $m, z_{0}=o$ and $\prod_{i=0}^{-1} A_{i}=1$.

Proof. For $n=0$, the result holds. Now suppose that $n>0$ and that our assumption holds for $n-1$. Then we have

$$
\begin{aligned}
& x_{12 n-7}=\frac{k(1+4 c f k o)}{(1+c f k o)(1+5 c f k o)} \prod_{i=0}^{n-2} \frac{(1+(12 i+8) c f k o)(1+(12 i+12) c f k o)(1+(12 i+16) c f k o)}{(1+(12 i+9) c f k o)(1+(12 i+13) c f k o)(1+(12 i+17) c f k o)}, \\
& x_{12 n-6}=\frac{l(1+a d g l)(1+5 a d g l)}{(1+2 a d g l)(1+6 a d g l)} \prod_{i=0}^{n-2} \frac{(1+(12 i+9) a d g l)(1+(12 i+13) a d g l)(1+(12 i+17) a d g l)}{(1+(12 i+10) a d g l)(1+(12 i+14) a d g l)(1+(12 i+18) a d g l)}, \\
& x_{12 n-5}=\frac{m(1+2 b e h m)(1+6 b e h m)}{(1+3 b e h m)(1+7 b e h m)} \prod_{i=0}^{n-2} \frac{(1+(12 i+10) b e h m)(1+(12 i+14) b e h m)(1+(12 i+18) b e h m)}{(1+(12 i+11) b e h m)(1+(12 i+15) b e h m)(1+(12 i+19) b e h m)}, \\
& x_{12 n-4}=\frac{o(1+3 c f k o)(1+7 c f k o)}{(1+4 c f k o)(1+8 c f k o)} \prod_{i=0}^{n-2} \frac{(1+(12 i+11) c f k o)(1+(12 i+15) c f k o)(1+(12 i+19) c f k o)}{(1+(12 i+12) c f k o)(1+(12 i+16) c f k o)(1+(12 i+20) c f k o)}, \\
& y_{12 n-7}=\frac{a(1+4 a d g l)}{(1+a d g l)(1+5 a d g l)} \prod_{i=0}^{n-2} \frac{(1+(12 i+8) a d g l)(1+(12 i+12) a d g l)(1+(12 i+16) a d g l)}{(1+(12 i+9) a d g l)(1+(12 i+13) a d g l)(1+(12 i+17) a d g l)},
\end{aligned}
$$




$$
\begin{aligned}
& y_{12 n-6}=\frac{b(1+b e h m)(1+5 b e h m)}{(1+2 b e h m)(1+6 b e h m)} \prod_{i=0}^{n-2} \frac{(1+(12 i+9) \text { behm })(1+(12 i+13) \text { behm })(1+(12 i+17) \text { behm })}{(1+(12 i+10) \text { behm })(1+(12 i+14) \text { behm })(1+(12 i+18) b e h m)}, \\
& y_{12 n-5}=\frac{c(1+2 c f k o)(1+6 c f k o)}{(1+3 c f k o)(1+7 c f k o)} \prod_{i=0}^{n-2} \frac{(1+(12 i+10) c f k o)(1+(12 i+14) c f k o)(1+(12 i+18) c f k o)}{(1+(12 i+11) c f k o)(1+(12 i+15) c f k o)(1+(12 i+19) c f k o)}, \\
& y_{12 n-4}=\frac{d(1+3 a d g l)(1+7 a d g l)}{(1+4 a d g l)(1+8 a d g l)} \prod_{i=0}^{n-2} \frac{(1+(12 i+11) a d g l)(1+(12 i+15) a d g l)(1+(12 i+19) a d g l)}{(1+(12 i+12) a d g l)(1+(12 i+16) a d g l)(1+(12 i+20) a d g l)}, \\
& z_{12 n-7}=\frac{e(1+4 b e h m)}{(1+b e h m)(1+5 b e h m)} \prod_{i=0}^{n-2} \frac{(1+(12 i+8) b e h m)(1+(12 i+12) b e h m)(1+(12 i+16) b e h m)}{(1+(12 i+9) b e h m)(1+(12 i+13) b e h m)(1+(12 i+17) b e h m)}, \\
& z_{12 n-6}=\frac{f(1+c f k o)(1+5 c f k o)}{(1+2 c f k o)(1+6 c f k o)} \prod_{i=0}^{n-2} \frac{(1+(12 i+9) c f k o)(1+(12 i+13) c f k o)(1+(12 i+17) c f k o)}{(1+(12 i+10) c f k o)(1+(12 i+14) c f k o)(1+(12 i+18) c f k o)}, \\
& z_{12 n-5}=\frac{g(1+2 a d g l)(1+6 a d g l)}{(1+3 a d g l)(1+7 a d g l)} \prod_{i=0}^{n-2} \frac{(1+(12 i+10) a d g l)(1+(12 i+14) a d g l)(1+(12 i+18) a d g l)}{(1+(12 i+11) a d g l)(1+(12 i+15) a d g l)(1+(12 i+19) a d g l)}, \\
& z_{12 n-4}=\frac{h(1+3 b e h m)(1+7 b e h m)}{(1+4 b e h m)(1+8 b e h m)} \prod_{i=0}^{n-2} \frac{(1+(12 i+11) b e h m)(1+(12 i+15) b e h m)(1+(12 i+19) b e h m)}{(1+(12 i+12) b e h m)(1+(12 i+16) b e h m)(1+(12 i+20) b e h m)} .
\end{aligned}
$$

Now, it follows from Eq. (2.1) that

$$
\begin{aligned}
& x_{12 n-3}=\frac{y_{12 n-7}}{1+y_{12 n-7} z_{12 n-5} x_{12 n-6} y_{12 n-4}} \\
& =\frac{\left(\frac{a(1+4 a d g l)}{(1+a d g l)(1+5 a d g l)} \prod_{i=0}^{n-2} \frac{(1+(12 i+8) a d g l)(1+(12 i+12) a d g l)(1+(12 i+16) a d g l)}{(1+(12 i+9) a d g l)(1+(12 i+13) a d g l)(1+(12 i+17) a d g l)}\right)}{\left(\begin{array}{c}
1+\frac{a(1+4 a d g l)}{(1+a d g l)(1+5 a d g l)} \prod_{i=0}^{n-2} \frac{(1+(12 i+8) a d g l)(1+(12 i+12) a d g l)(1+(12 i+16) a d g l)}{(1+(12 i+9) a d g l)(1+(12 i+13) a d g l)(1+(12 i+17) a d g l)} \\
\frac{g(1+2 a d g l)(1+6 a d g l)}{(1+3 a d g l)(1+7 a d g l)} \prod_{i=0}^{n-2} \frac{(1+(12 i+10) a d g l)(1+(12 i+14) a d g l)(1+(12 i+18) a d g l)}{(1+(12 i+11) a d g l)(1+(12 i+15) a d g l)(1+(12 i+19) a d g l)} \\
\frac{l(1+a d g l)(1+5 a d g l)}{(1+2 a d g l)(1+6 a d g l)} \prod_{i=0}^{n-2} \frac{(1+(12 i+9) a d g l)(1+(12 i+13) a d g l)(1+(12 i+17) a d g l)}{(1+(12 i+10) a d g l)(1+(12 i+14) a d g l)(1+(12 i+18) a d g l)} \\
\frac{d(1+3 a d g l)(1+7 a d g l)}{(1+4 a d g l)(1+8 a d g l)} \prod_{i=0}^{n-2} \frac{(1+(12 i+11) a d g l)(1+(12 i+15) a d g l)(1+(12 i+19) a d g l)}{(1+(12 i+12) a d g l)(1+(12 i+16) a d g l)(1+(12 i+20) a d g l)}
\end{array}\right)} \\
& =\frac{a(1+4 a d g l)(1+8 a d g l)\left(\prod_{i=0}^{n-2}(1+(12 i+8) a d g l)(1+(12 i+12) a d g l)(1+(12 i+16) a d g l)\right)}{(1+a d g l)(1+5 a d g l)\left(\prod_{i=0}^{n-2}(1+(12 i+9) a d g l)(1+(12 i+13) a d g l)(1+(12 i+17) a d g l)\right)} \\
& \times \frac{\left(\prod_{i=0}^{n-2}(1+(12 i+20) a d g l)\right)}{(1+8 a d g l)\left(\prod_{i=0}^{n-2}(1+(12 i+20) a d g l)\right)+a d g l\left(\prod_{i=0}^{n-2}(1+(12 i+8) a d g l)\right)} \\
& =\frac{a(1+4 a d g l)(1+8 a d g l)\left(\prod_{i=0}^{n-2}(1+(12 i+12) a d g l)(1+(12 i+16) a d g l)(1+(12 i+20) a d g l)\right)}{(1+a d g l)(1+5 a d g l)\left(\prod_{i=0}^{n-2}(1+(12 i+9) a d g l)(1+(12 i+13) a d g l)(1+(12 i+17) a d g l)\right)} \frac{1}{(1+(12 n-4) a d g l)+a d g l} \\
& =\frac{\left(a(1+4 a d g l)(1+8 a d g l)\left(\prod_{i=0}^{n-2}(1+(12 i+12) a d g l)(1+(12 i+16) a d g l)(1+(12 i+20) a d g l)\right)\right)}{\left((1+a d g l)(1+5 a d g l)(1+(12 n-3) a d g l)\left(\prod_{i=0}^{n-2}(1+(12 i+9) a d g l)(1+(12 i+13) a d g l)(1+(12 i+17) a d g l)\right)\right)} \\
& =a \prod_{i=0}^{n-1} \frac{(1+12 i a d g l)(1+(12 i+4) a d g l)(1+(12 i+8) a d g l)}{(1+(12 i+1) a d g l)(1+(12 i+5) a d g l)(1+(12 i+9) a d g l)} \text {. }
\end{aligned}
$$


Also, we see that

$$
\begin{aligned}
& y_{4 n-2}=\frac{z_{12 n-7}}{1+z_{12 n-7} x_{12 n-5} y_{12 n-6} z_{12 n-4}},
\end{aligned}
$$

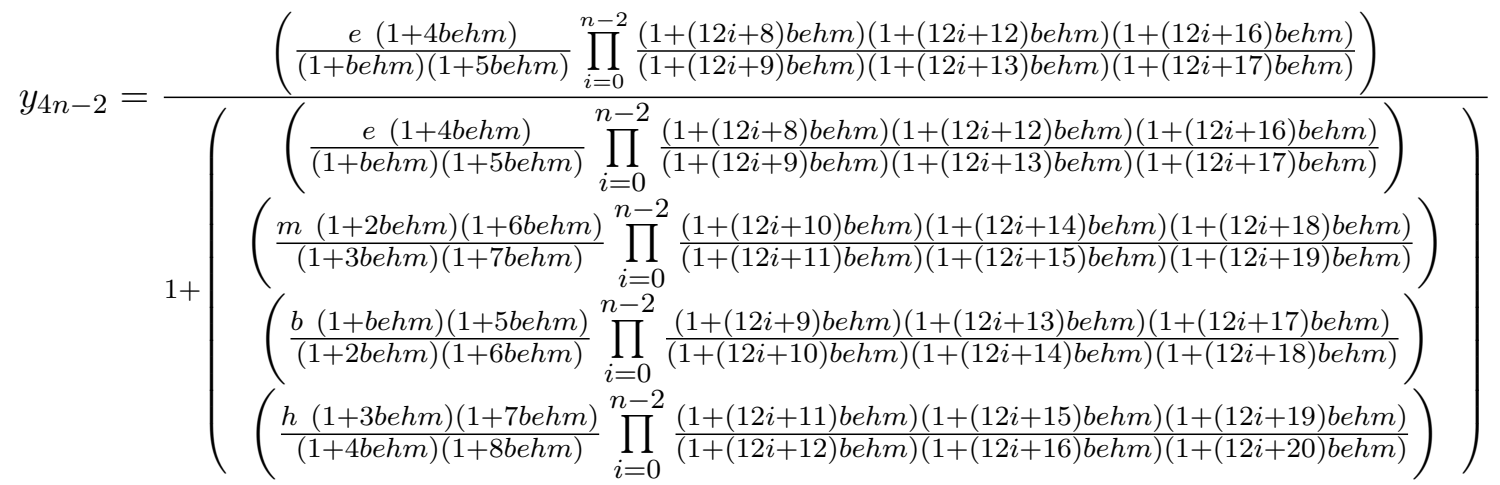

$$
\begin{aligned}
& =\frac{(e(1+4 b e h m)(1+8 b e h m))\left(\prod_{i=0}^{n-2}(1+(12 i+12) \text { behm })(1+(12 i+16) b e h m)(1+(12 i+20) \text { behm })\right)}{(1+\text { behm })(1+5 \text { behm })\left(\prod_{i=0}^{n-2}(1+(12 i+9) \text { behm })(1+(12 i+13) \text { behm })(1+(12 i+17) b e h m)\right)} \\
& \times \frac{\left(\prod_{i=0}^{n-2}(1+(12 i+8) b e h m)\right)}{((1+8 b e h m))\left(\prod_{i=0}^{n-2}(1+(12 i+20) b e h m)\right)+b e h m\left(\prod_{i=0}^{n-2}(1+(12 i+8) b e h m)\right)} \\
& =\frac{(e(1+4 b e h m)(1+8 b e h m))\left(\prod_{i=0}^{n-2}(1+(12 i+12) b e h m)(1+(12 i+16) b e h m)(1+(12 i+20) b e h m)\right)}{(1+\text { behm })(1+5 b e h m)\left(\prod_{i=0}^{n-2}(1+(12 i+9) b e h m)(1+(12 i+13) b e h m)(1+(12 i+17) b e h m)\right)} \\
& \times \frac{\left(\prod_{i=0}^{n-2}(1+(12 i+8) b e h m)\right)}{\left(\prod_{i=0}^{n-1}(1+(12 i+8) b e h m)\right)+b e h m\left(\prod_{i=0}^{n-2}(1+(12 i+8) b e h m)\right)} \\
& =\frac{(e(1+4 b e h m)(1+8 b e h m))\left(\prod_{i=0}^{n-2}(1+(12 i+12) b e h m)(1+(12 i+16) b e h m)(1+(12 i+20) b e h m)\right)}{(1+b e h m)(1+5 b e h m)\left(\prod_{i=0}^{n-2}(1+(12 i+9) b e h m)(1+(12 i+13) b e h m)(1+(12 i+17) b e h m)\right)} \\
& \times \frac{\left(\prod_{i=0}^{n-2}(1+(12 i+8) \text { behm })\right)}{\left(\prod_{i=0}^{n-2}(1+(12 i+8) \text { behm })\right)((1+(12 n-4) \text { behm })+\text { behm })} \\
& =\frac{(e(1+4 b e h m)(1+8 b e h m))\left(\prod_{i=0}^{n-2}(1+(12 i+12) b e h m)(1+(12 i+16) b e h m)(1+(12 i+20) b e h m)\right)}{(1+\text { behm })(1+5 b e h m)(1+(12 n-3) b e h m)\left(\prod_{i=0}^{n-2}(1+(12 i+9) b e h m)(1+(12 i+13) b e h m)(1+(12 i+17) b e h m)\right)} \\
& =e \prod_{i=0}^{n-1} \frac{(1+(12 i) b e h m)(1+(12 i+4) \text { behm })(1+(12 i+8) \text { behm })}{(1+(12 i+1) b e h m)(1+(12 i+5) b e h m)(1+(12 i+9) b e h m)},
\end{aligned}
$$

and

$$
z_{12 n-3}=\frac{x_{12 n-7}}{1+x_{12 n-7} y_{12 n-5} z_{12 n-6} x_{12 n-4}}
$$




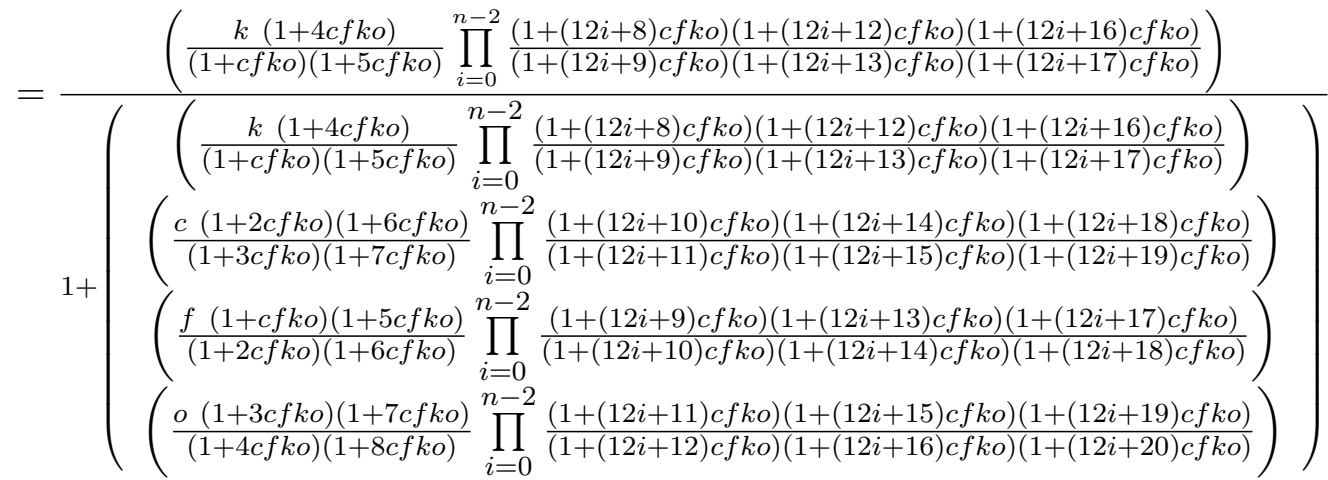

$$
\begin{aligned}
& =\frac{(k(1+4 c f k o)(1+8 c f k o))\left(\prod_{i=0}^{n-2}(1+(12 i+12) c f k o)(1+(12 i+16) c f k o)(1+(12 i+20) c f k o)\right)}{(1+c f k o)(1+5 c f k o)\left(\prod_{i=0}^{n-2}(1+(12 i+9) c f k o)(1+(12 i+13) c f k o)(1+(12 i+17) c f k o)\right)} \\
& \times \frac{\left(\prod_{i=0}^{n-2}(1+(12 i+8) c f k o)\right)}{\left(\prod_{i=0}^{n-1}(1+(12 i+8) c f k o)\right)+(c f k o)\left(\prod_{i=0}^{n-2}(1+(12 i+8) c f k o)\right)} \\
& =\frac{(k(1+4 c f k o)(1+8 c f k o))\left(\prod_{i=0}^{n-2}(1+(12 i+12) c f k o)(1+(12 i+16) c f k o)(1+(12 i+20) c f k o)\right)}{(1+c f k o)(1+5 c f k o)\left(\prod_{i=0}^{n-2}(1+(12 i+9) c f k o)(1+(12 i+13) c f k o)(1+(12 i+17) c f k o)\right)} \\
& \times \frac{\left(\prod_{i=0}^{n-2}(1+(12 i+8) c f k o)\right)}{(1+(12 n-4) c f k o)\left(\prod_{i=0}^{n-2}(1+(12 i+8) c f k o)\right)+(c f k o)\left(\prod_{i=0}^{n-2}(1+(12 i+8) c f k o)\right)} \\
& =\frac{(k(1+4 c f k o)(1+8 c f k o))\left(\prod_{i=0}^{n-2}(1+(12 i+12) c f k o)(1+(12 i+16) c f k o)(1+(12 i+20) c f k o)\right)}{(1+c f k o)(1+5 c f k o)\left(\prod_{i=0}^{n-2}(1+(12 i+9) c f k o)(1+(12 i+13) c f k o)(1+(12 i+17) c f k o)\right)} \\
& \times \frac{\left(\prod_{i=0}^{n-2}(1+(12 i+8) c f k o)\right)}{\left(\prod_{i=0}^{n-2}(1+(12 i+8) c f k o)\right)(1+(12 n-4) c f k o+c f k o)} \\
& =\frac{(k(1+4 c f k o)(1+8 c f k o))\left(\prod_{i=0}^{n-2}(1+(12 i+12) c f k o)(1+(12 i+16) c f k o)(1+(12 i+20) c f k o)\right)}{(1+c f k o)(1+5 c f k o)(1+(12 n-3) c f k o)\left(\prod_{i=0}^{n-2}(1+(12 i+9) c f k o)(1+(12 i+13) c f k o)(1+(12 i+17) c f k o)\right)}, \\
& z_{12 n-3}=\frac{k \prod_{i=0}^{n-1}(1+(12 i) c f k o)(1+(12 i+4) c f k o)(1+(12 i+8) c f k o)}{\prod_{i=0}^{n-1}(1+(12 i+1) c f k o)(1+(12 i+5) c f k o)(1+(12 i+9) c f k o)} .
\end{aligned}
$$

Also, we can prove the other relations. This completes the proof.

Lemma 2.2. Let $\left\{x_{n}, y_{n}, z_{n}\right\}$ be positive solutions of system (2.1), then $\left\{x_{n}\right\},\left\{y_{n}\right\}$ and $\left\{z_{n}\right\}$ are bounded and converges to zero.

Proof. It follows from Eq. 2.1p that

$$
x_{n+1}=\frac{y_{n-3}}{1+y_{n} z_{n-1} x_{n-2} y_{n-3}}<y_{n-3}, y_{n+1}=\frac{z_{n-3}}{1+z_{n} x_{n-1} y_{n-2} z_{n-3}}<z_{n-3},
$$




$$
z_{n+1}=\frac{x_{n-3}}{1+x_{n} y_{n-1} z_{n-2} x_{n-3}}<x_{n-3} .
$$

Thus

$$
\begin{aligned}
x_{n+5} & <y_{n+1}, y_{n+5}<z_{n+1}, z_{n+5}<x_{n+1} \Rightarrow x_{n+5}<z_{n-3}, y_{n+5}<x_{n-3}, z_{n+5}<y_{n-3} \\
& \Rightarrow x_{n+9}<y_{n+5}<x_{n-3}, y_{n+9}<z_{n+5}<y_{n-3}, \quad z_{n+9}<x_{n+5}<z_{n-3} .
\end{aligned}
$$

Then the subsequences $\left\{x_{12 n+i}\right\}_{n=0}^{\infty}, i=-3 .-2,-1,0,1,2, \ldots, 8$, are decreasing and bounded from above by $M=\max \left\{x_{-3}, x_{-2}, x_{-1}, x_{0}, \ldots, x_{8}\right\}$. Also, the subsequences $\left\{y_{12 n+i}\right\}_{n=0}^{\infty}$ and $\left\{z_{12 n+i}\right\}_{n=0}^{\infty}, i=$ $-3 .-2,-1,0,1,2, \ldots, 8$, are decreasing and bounded from above by $L=\max \left\{y_{-3}, y_{-2}, \ldots, y_{8}\right\}$ and $N=$ $\max \left\{z_{-3}, z_{-2}, \ldots, z_{8}\right\}$, respectively. This completes the proof.

Lemma 2.3. If $x_{i}, y_{i}, z_{i}, i=-3,-2,-1,0$, are arbitrary real numbers and let $\left\{x_{n}, y_{n}, z_{n}\right\}$ be solutions of system (2.1), then the following statements are true.

(i) If $x_{-3}=a=0$, then we have $x_{12 n-3}=y_{12 n+5}=z_{12 n+1}=0, x_{12 n}=y_{12 n+8}=z_{12 n+4}=d, x_{12 n+6}=$ $y_{12 n+2}=z_{12 n-2}=l$ and $x_{12 n+3}=y_{12 n-1}=z_{12 n+7}=g$.

(ii) If $x_{-2}=b=0$, then we have $x_{12 n-2}=y_{12 n+6}=z_{12 n+2}=0, x_{12 n+1}=y_{12 n-3}=z_{12 n+5}=e$, $x_{12 n+4}=y_{12 n}=z_{12 n+8}=h$ and $x_{12 n+7}=y_{12 n+3}=z_{12 n-1}=m$.

(iii) If $x_{-1}=c=0$, then we have $x_{12 n-1}=y_{12 n+7}=z_{12 n+3}=0, x_{12 n+2}=y_{12 n-2}=z_{12 n+6}=f$, $x_{12 n+5}=y_{12 n+1}=z_{12 n-3}=k$ and $x_{12 n+8}=y_{12 n+4}=z_{12 n}=o$.

(iv) If $x_{0}=d=0$, then we have $x_{12 n}=y_{12 n+8}=z_{12 n+4}=0, x_{12 n-3}=y_{12 n+5}=z_{12 n+1}=a, x_{12 n+3}=$ $y_{12 n-1}=z_{12 n+7}=g$ and $x_{12 n+6}=y_{12 n+2}=z_{12 n-2}=l$.

(v) If $y_{-3}=e=0$, then we have $x_{12 n+1}=y_{12 n-3}=z_{12 n+5}=0, x_{12 n+7}=y_{12 n+3}=z_{12 n-1}=m$, $x_{12 n-2}=y_{12 n+6}=z_{12 n+2}=b$ and $x_{12 n+4}=y_{12 n}=z_{12 n+8}=h$.

(vi) If $y_{-2}=f=0$, then we have $x_{12 n+2}=y_{12 n-2}=z_{12 n+6}=0, x_{12 n+5}=y_{12 n+1}=z_{12 n-3}=k$, $x_{12 n+8}=y_{12 n+4}=z_{12 n}=o$ and $x_{12 n+7}=y_{12 n+3}=z_{12 n-1}=m$.

(vii) If $y_{-1}=g=0$, then we have $x_{12 n+3}=y_{12 n-1}=z_{12 n+7}=0, x_{12 n+6}=y_{12 n+2}=z_{12 n-2}=l$, $x_{12 n-3}=y_{12 n+5}=z_{12 n+1}=a$ and $x_{12 n}=y_{12 n+8}=z_{12 n+4}=d$.

(viii) If $y_{0}=h=0$, then we have $x_{12 n+4}=y_{12 n}=z_{12 n+8}=0, x_{12 n+7}=y_{12 n+3}=z_{12 n-1}=m, x_{12 n-2}=$ $y_{12 n+6}=z_{12 n+2}=b$ and $x_{12 n+1}=y_{12 n-3}=z_{12 n+5}=e$.

(ix) If $z_{-3}=k=0$, then we have $x_{12 n+5}=y_{12 n+1}=z_{12 n-3}=0, x_{12 n+2}=y_{12 n-2}=z_{12 n+6}=f$, $x_{12 n+8}=y_{12 n+4}=z_{12 n}=o$ and $x_{12 n-1}=y_{12 n+7}=z_{12 n+3}=c$.

(x) If $z_{-2}=l=0$, then we have $x_{12 n+6}=y_{12 n+2}=z_{12 n-2}=0, x_{12 n+3}=y_{12 n-1}=z_{12 n+7}=g$, $x_{12 n-3}=y_{12 n+5}=z_{12 n+1}=a$ and $x_{12 n}=y_{12 n+8}=z_{12 n+4}=d$.

(xi) If $z_{-1}=m=0$, then we have $x_{12 n+7}=y_{12 n+3}=z_{12 n-1}=0, x_{12 n+1}=y_{12 n-3}=z_{12 n+5}=e$, $x_{12 n+4}=y_{12 n}=z_{12 n+8}=h$ and $x_{12 n-2}=y_{12 n+6}=z_{12 n+2}=b$.

(xii) If $z_{0}=o=0$, then we have $x_{12 n+8}=y_{12 n+4}=z_{12 n}=0$ and $x_{12 n+2}=y_{12 n-2}=z_{12 n+6}=f$, $x_{12 n+5}=y_{12 n+1}=z_{12 n-3}=k, x_{12 n-1}=y_{12 n+7}=z_{12 n+3}=c$.

Proof. The proof follows from the form of the solutions of system 2.1). 
Theorem 2.4. The solutions of the system

$$
x_{n+1}=\frac{y_{n-3}}{1-y_{n-3} z_{n-1} x_{n-2} y_{n}}, y_{n+1}=\frac{z_{n-3}}{1-z_{n-3} x_{n-1} y_{n-2} z_{n}}, z_{n+1}=\frac{x_{n-3}}{1-x_{n-3} y_{n-1} z_{n-2} x_{n}},
$$

are given by the following equations

$$
\begin{aligned}
& x_{12 n-3}=(-1)^{n} a \prod_{i=0}^{n-1} \frac{(-1+12 i a d g l)(-1+(12 i+4) \text { adgl })(-1+(12 i+8) \text { adgl })}{(-1+(12 i+1) a d g l)(-1+(12 i+5) a d g l)(-1+(12 i+9) a d g l)}, \\
& x_{12 n-2}=b \prod_{i=0}^{n-1} \frac{(-1+(12 i+1) \text { behm })(-1+(12 i+5) b e h m)(-1+(12 i+9) b e h m)}{(-1+(12 i+2) b e h m)(-1+(12 i+6) b e h m)(-1+(12 i+10) b e h m)}, \\
& x_{12 n-1}=c \prod_{i=0}^{n-1} \frac{(-1+(12 i+2) c f k o)(-1+(12 i+6) c f k o)(-1+(12 i+10) c f k o)}{(-1+(12 i+3) c f k o)(-1+(12 i+7) c f k o)(-1+(12 i+11) c f k o)}, \\
& x_{12 n}=d \prod_{i=0}^{n-1} \frac{(-1+(12 i+3) a d g l)(-1+(12 i+7) a d g l)(-1+(12 i+11) a d g l)}{(-1+(12 i+4) a d g l)(-1+(12 i+8) a d g l)(-1+(12 i+12) a d g l)}, \\
& x_{12 n+1}=-\frac{e}{(-1+b e h m)} \prod_{i=0}^{n-1} \frac{(-1+(12 i+4) b e h m)(-1+(12 i+8) \text { behm })(-1+(12 i+12) b e h m)}{(-1+(12 i+5) b e h m)(-1+(12 i+9) \text { behm })(-1+(12 i+13) b e h m)}, \\
& x_{12 n+2}=\frac{f(-1+c f k o)}{(-1+2 c f k o)} \prod_{i=0}^{n-1} \frac{(-1+(12 i+5) c f k o)(-1+(12 i+9) c f k o)(-1+(12 i+13) c f k o)}{(-1+(12 i+6) c f k o)(-1+(12 i+10) c f k o)(-1+(12 i+14) c f k o)}, \\
& x_{12 n+3}=\frac{g(-1+2 a d g l)}{(-1+3 a d g l)} \prod_{i=0}^{n-1} \frac{(-1+(12 i+6) \text { adgl })(-1+(12 i+10) \text { adgl })(-1+(12 i+14) a d g l)}{(-1+(12 i+7) a d g l)(-1+(12 i+11) a d g l)(-1+(12 i+15) a d g l)}, \\
& x_{12 n+4}=\frac{h(-1+3 b e h m)}{(-1+4 b e h m)} \prod_{i=0}^{n-1} \frac{(-1+(12 i+7) \text { behm })(-1+(12 i+11) b e h m)(-1+(12 i+15) \text { behm })}{(-1+(12 i+8) \text { behm })(-1+(12 i+12) \text { behm })(-1+(12 i+16) \text { behm })}, \\
& x_{12 n+5}=-\frac{k(-1+4 c f k o)}{(-1+c f k o)(-1+5 c f k o)} \prod_{i=0}^{n-1} \frac{(-1+(12 i+8) c f k o)(-1+(12 i+12) c f k o)(-1+(12 i+16) c f k o)}{(-1+(12 i+9) c f k o)(-1+(12 i+13) c f k o)(-1+(12 i+17) c f k o)}, \\
& x_{12 n+6}=\frac{l(-1+\text { adgl })(-1+5 a d g l)}{(-1+2 a d g l)(-1+6 a d g l)} \prod_{i=0}^{n-1} \frac{(-1+(12 i+9) \text { adgl })(-1+(12 i+13) \text { adgl })(-1+(12 i+17) \text { adgl })}{(-1+(12 i+10) \text { adgl })(-1+(12 i+14) a d g l)(-1+(12 i+18) a d g l)}, \\
& x_{12 n+7}=\frac{m(-1+2 b e h m)(-1+6 b e h m)}{(-1+3 b e h m)(-1+7 b e h m)} \prod_{i=0}^{n-1} \frac{(-1+(12 i+10) \text { behm })(-1+(12 i+14) \text { behm })(-1+(12 i+18) \text { behm })}{(-1+(12 i+11) \text { behm })(-1+(12 i+15) \text { behm })(-1+(12 i+19) \text { behm })}, \\
& x_{12 n+8}=\frac{o(-1+3 c f k o)(-1+7 c f k o)}{(-1+4 c f k o)(-1+8 c f k o)} \prod_{i=0}^{n-1} \frac{(-1+(12 i+11) c f k o)(-1+(12 i+15) c f k o)(-1+(12 i+19) c f k o)}{(-1+(12 i+12) c f k o)(-1+(12 i+16) c f k o)(-1+(12 i+20) c f k o)}, \\
& y_{12 n-3}=(-1)^{n} e \prod_{i=0}^{n-1} \frac{(-1+(12 i) b e h m)(-1+(12 i+4) \text { behm })(-1+(12 i+8) \text { behm })}{(-1+(12 i+1) b e h m)(-1+(12 i+5) \text { behm })(-1+(12 i+9) b e h m)}, \\
& y_{12 n-2}=f \prod_{i=0}^{n-1} \frac{(-1+(12 i+1) c f k o)(-1+(12 i+5) c f k o)(-1+(12 i+9) c f k o)}{(-1+(12 i+2) c f k o)(-1+(12 i+6) c f k o)(-1+(12 i+10) c f k o)}, \\
& y_{12 n-1}=g \prod_{i=0}^{n-1} \frac{(-1+(12 i+2) a d g l)(1+(12 i+6) a d g l)(-1+(12 i+10) a d g l)}{(-1+(12 i+3) a d g l)(-1+(12 i+7) a d g l)(-1+(12 i+11) a d g l)}, \\
& y_{12 n}=h \prod_{i=0}^{n-1} \frac{(-1+(12 i+3) \text { behm })(-1+(12 i+7) b e h m)(-1+(12 i+11) b e h m)}{(-1+(12 i+4) \text { behm })(-1+(12 i+8) b e h m)(-1+(12 i+12) b e h m)}, \\
& y_{12 n+1}=-\frac{k}{(-1+c f k o)} \prod_{i=0}^{n-1} \frac{(-1+(12 i+4) c f k o)(-1+(12 i+8) c f k o)(-1+(12 i+12) c f k o)}{(-1+(12 i+5) c f k o)(-1+(12 i+9) c f k o)(-1+(12 i+13) c f k o)}, \\
& y_{12 n+2}=\frac{l(-1+a d g l)}{(-1+2 a d g l)} \prod_{i=0}^{n-1} \frac{(-1+(12 i+5) a d g l)(-1+(12 i+9) a d g l)(-1+(12 i+13) a d g l)}{(-1+(12 i+6) a d g l)(-1+(12 i+10) a d g l)(-1+(12 i+14) a d g l)}, \\
& y_{12 n+3}=\frac{m(-1+2 b e h m)}{(-1+3 b e h m)} \prod_{i=0}^{n-1} \frac{(-1+(12 i+6) b e h m)(-1+(12 i+10) b e h m)(-1+(12 i+14) b e h m)}{(-1+(12 i+7) b e h m)(-1+(12 i+11) b e h m)(-1+(12 i+15) b e h m)}, \\
& y_{12 n+4}=\frac{o(-1+3 c f k o)}{(-1+4 c f k o)} \prod_{i=0}^{n-1} \frac{(-1+(12 i+7) c f k o)(-1+(12 i+11) c f k o)(-1+(12 i+15) c f k o)}{(-1+(12 i+8) c f k o)(-1+(12 i+12) c f k o)(-1+(12 i+16) c f k o)},
\end{aligned}
$$




$$
\begin{aligned}
& y_{12 n+5}=-\frac{a(-1+4 a d g l)}{(-1+a d g l)(-1+5 a d g l)} \prod_{i=0}^{n-1} \frac{(-1+(12 i+8) a d g l)(-1+(12 i+12) a d g l)(-1+(12 i+16) a d g l)}{(-1+(12 i+9) a d g l)(-1+(12 i+13) a d g l)(-1+(12 i+17) a d g l)}, \\
& y_{12 n+6}=\frac{b(-1+\text { behm })(-1+5 \text { behm })}{(-1+2 b e h m)(-1+6 b e h m)} \prod_{i=0}^{n-1} \frac{(-1+(12 i+9) \text { behm })(-1+(12 i+13) \text { behm })(-1+(12 i+17) \text { behm })}{(-1+(12 i+10) \text { behm })(-1+(12 i+14) \text { behm })(-1+(12 i+18) \text { behm })}, \\
& y_{12 n+7}=\frac{c(-1+2 c f k o)(-1+6 c f k o)}{(-1+3 c f k o)(-1+7 c f k o)} \prod_{i=0}^{n-1} \frac{(-1+(12 i+10) c f k o)(-1+(12 i+14) c f k o)(-1+(12 i+18) c f k o)}{(-1+(12 i+11) c f k o)(-1+(12 i+15) c f k o)(-1+(12 i+19) c f k o)}, \\
& y_{12 n+8}=\frac{d(-1+3 a d g l)(-1+7 a d g l)}{(-1+4 a d g l)(-1+8 a d g l)} \prod_{i=0}^{n-1} \frac{(-1+(12 i+11) a d g l)(-1+(12 i+15) a d g l)(-1+(12 i+19) a d g l)}{(-1+(12 i+12) a d g l)(-1+(12 i+16) a d g l)(-1+(12 i+20) a d g l)}, \\
& z_{12 n-3}=(-1)^{n} k \prod_{i=0}^{n-1} \frac{(-1+(12 i) c f k o)(-1+(12 i+4) c f k o)(-1+(12 i+8) c f k o)}{(-1+(12 i+1) c f k o)(-1+(12 i+5) c f k o)(-1+(12 i+9) c f k o)}, \\
& z_{12 n-2}=l \prod_{i=0}^{n-1} \frac{(-1+(12 i+1) a d g l)(-1+(12 i+5) a d g l)(-1+(12 i+9) a d g l)}{(-1+(12 i+2) a d g l)(-1+(12 i+6) a d g l)(-1+(12 i+10) a d g l)}, \\
& z_{12 n-1}=m \prod_{i=0}^{n-1} \frac{(-1+(12 i+2) \text { behm })(-1+(12 i+6) \text { behm })(-1+(12 i+10) \text { behm })}{(-1+(12 i+3) \text { behm })(-1+(12 i+7) \text { behm })(-1+(12 i+11) \text { behm })}, \\
& z_{12 n}=o \prod_{i=0}^{n-1} \frac{(-1+(12 i+3) c f k o)(-1+(12 i+7) c f k o)(-1+(12 i+11) c f k o)}{(-1+(12 i+4) c f k o)(-1+(12 i+8) c f k o)(-1+(12 i+12) c f k o)} \\
& z_{12 n+1}=-\frac{a}{(-1+a d g l)} \prod_{i=0}^{n-1} \frac{(-1+(12 i+4) a d g l)(-1+(12 i+8) a d g l)(-1+(12 i+12) a d g l)}{(-1+(12 i+5) a d g l)(-1+(12 i+9) a d g l)(-1+(12 i+13) a d g l)}, \\
& z_{12 n+2}=\frac{b(-1+\text { behm })}{(-1+2 b e h m)} \prod_{i=0}^{n-1} \frac{(-1+(12 i+5) \text { behm })(-1+(12 i+9) \text { behm })(-1+(12 i+13) \text { behm })}{(-1+(12 i+6) \text { behm })(-1+(12 i+10) \text { behm })(-1+(12 i+14) \text { behm })}, \\
& z_{12 n+3}=\frac{c(-1+2 c f k o)}{(-1+3 c f k o)} \prod_{i=0}^{n-1} \frac{(-1+(12 i+6) c f k o)(-1+(12 i+10) c f k o)(-1+(12 i+14) c f k o)}{(-1+(12 i+7) c f k o)(-1+(12 i+11) c f k o)(-1+(12 i+15) c f k o)}, \\
& z_{12 n+4}=\frac{d(-1+3 a d g l)}{(-1+4 a d g l)} \prod_{i=0}^{n-1} \frac{(-1+(12 i+7) a d g l)(-1+(12 i+11) a d g l)(-1+(12 i+15) a d g l)}{(-1+(12 i+8) a d g l)(-1+(12 i+12) a d g l)(-1+(12 i+16) a d g l)}, \\
& z_{12 n+5}=-\frac{e(-1+4 b e h m)}{(-1+\text { behm })(-1+5 b e h m)} \prod_{i=0}^{n-1} \frac{(-1+(12 i+8) \text { behm })(-1+(12 i+12) \text { behm })(-1+(12 i+16) \text { behm })}{(-1+(12 i+9) \text { behm })(-1+(12 i+13) \text { behm })(-1+(12 i+17) \text { behm })}, \\
& z_{12 n+6}=\frac{f(-1+c f k o)(-1+5 c f k o)}{(-1+2 c f k o)(-1+6 c f k o)} \prod_{i=0}^{n-1} \frac{(-1+(12 i+9) c f k o)(-1+(12 i+13) c f k o)(-1+(12 i+17) c f k o)}{(-1+(12 i+10) c f k o)(-1+(12 i+14) c f k o)(-1+(12 i+18) c f k o)} \\
& z_{12 n+7}=\frac{g(-1+2 a d g l)(-1+6 a d g l)}{(-1+3 a d g l)(-1+7 a d g l)} \prod_{i=0}^{n-1} \frac{(-1+(12 i+10) a d g l)(-1+(12 i+14) a d g l)(-1+(12 i+18) a d g l)}{(-1+(12 i+11) a d g l)(-1+(12 i+15) a d g l)(-1+(12 i+19) a d g l)}, \\
& z_{12 n+8}=\frac{h(-1+3 b e h m)(-1+7 b e h m)}{(-1+4 b e h m)(-1+8 b e h m)} \prod_{i=0}^{n-1} \frac{(-1+(12 i+11) \text { behm })(-1+(12 i+15) \text { behm })(-1+(12 i+19) \text { behm })}{(-1+(12 i+12) \text { behm })(-1+(12 i+16) \text { behm })(-1+(12 i+20) \text { behm })},
\end{aligned}
$$

where $\prod_{i=0}^{-1} A_{i}=1$.

3. On the systems: $x_{n+1}=\frac{y_{n-3}}{ \pm 1+y_{n} z_{n-1} x_{n-2} y_{n-3}}, y_{n+1}=\frac{z_{n-3}}{ \pm 1-z_{n} x_{n-1} y_{n-2} z_{n-3}}, z_{n+1}=\frac{x_{n-3}}{ \pm 1-x_{n} y_{n-1} z_{n-2} x_{n-3}}$

In this section, we study the solutions of the system of three difference equations in the following form

$$
x_{n+1}=\frac{y_{n-3}}{1+y_{n} z_{n-1} x_{n-2} y_{n-3}}, y_{n+1}=\frac{z_{n-3}}{1-z_{n} x_{n-1} y_{n-2} z_{n-3}}, z_{n+1}=\frac{x_{n-3}}{-1-x_{n} y_{n-1} z_{n-2} x_{n-3}}, n=0,1, \ldots,
$$

with nonzero real initial conditions.

Theorem 3.1. Suppose that $\left\{x_{n}, y_{n}, z_{n}\right\}$ are solutions of system (3.1), we see that

$$
x_{12 n-3}=\frac{a(1+2 a d g l)^{n}}{(-1+a d g l)^{n}(1+a d g l)^{2 n}},
$$

$$
x_{12 n-2}=\frac{(-1)^{n} b(1+b e h m)^{2 n}(1+3 b e h m)^{n}}{(1+2 b e h m)^{2 n}},
$$




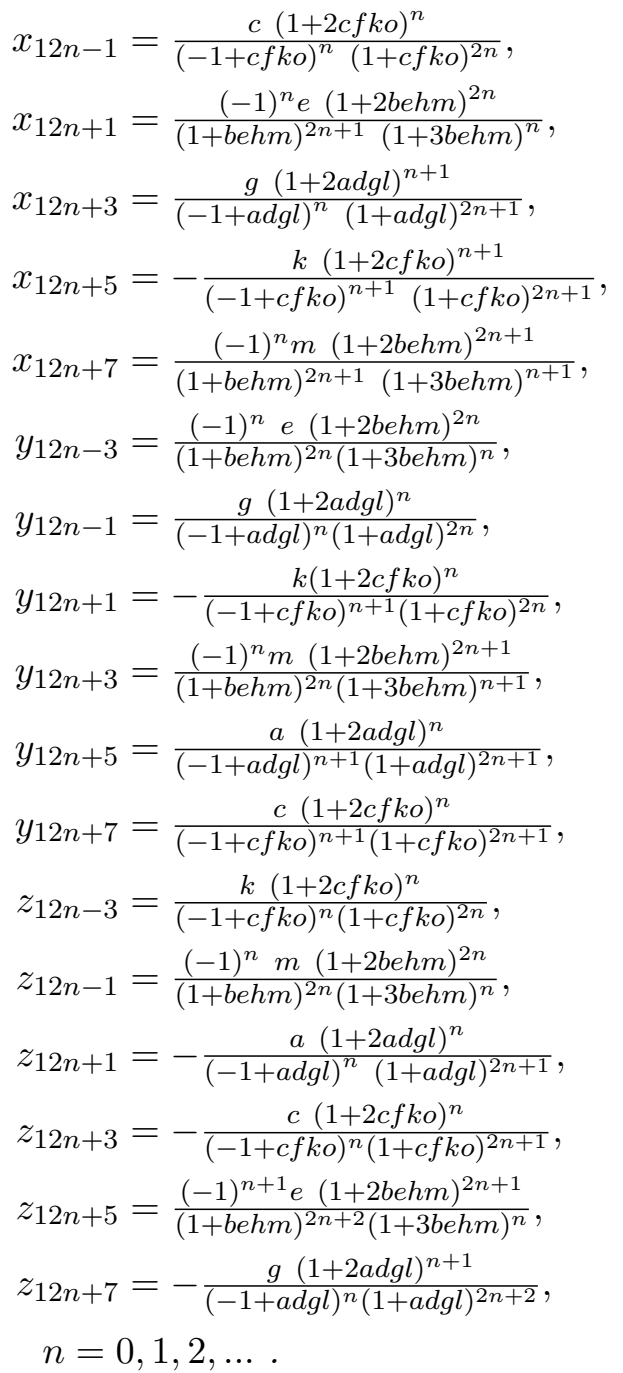

$$
\begin{aligned}
& x_{12 n}=\frac{d(-1+a d g l)^{n}(1+a d g l)^{2 n}}{(1+2 a d g l)^{n}}, \\
& x_{12 n+2}=-\frac{f(-1+c f k o)^{n+1}(1+c f k o)^{2 n}}{(1+2 c f k o)^{n}} \text {, } \\
& x_{12 n+4}=\frac{(-1)^{n} h(1+b e h m)^{2 n}(1+3 b e h m)^{n+1}}{(1+2 b e h m)^{2 n+1}} \text {, } \\
& x_{12 n+6}=-\frac{l(-1+a d g l)^{n+1}(1+a d g l)^{2 n+1}}{(1+2 a d g l)^{n+1}}, \\
& x_{12 n+8}=-\frac{o(-1+c f k o)^{n+1}(1+c f k o)^{2 n+1}}{(1+2 c f k o)^{n+1}}, \\
& y_{12 n-2}=\frac{f(-1+c f k o)^{n}(1+c f k o)^{2 n}}{(1+2 c f k o)^{n}}, \\
& y_{12 n}=\frac{(-1)^{n} h(1+b e h m)^{2 n}(1+3 b e h m)^{n}}{(1+2 b e h m)^{2 n}}, \\
& y_{12 n+2}=\frac{l(1+a d g l)^{2 n+1}(-1+a d g l)^{n}}{(1+2 a d g l)^{n+1}}, \\
& y_{12 n+4}=\frac{o(-1+c f k o)^{n}(1+c f k o)^{2 n+1}}{(1+2 c f k o)^{n+1}} \text {, } \\
& y_{12 n+6}=\frac{(-1)^{n+1} b(1+\text { behm })^{2 n+2}(1+3 b e h m)^{n}}{(1+2 b e h m)^{2 n+1}}, \\
& y_{12 n+8}=-\frac{d(-1+a d g l)^{n}(1+a d g l)^{2 n+2}}{(1+2 a d g l)^{n+1}}, \\
& z_{12 n-2}=\frac{l(-1+a d g l)^{n}(1+a d g l)^{2 n}}{(1+2 a d g l)^{n}}, \\
& z_{12 n}=\frac{o(-1+c f k o)^{n}(1+c f k o)^{2 n}}{(1+2 c f k o)^{n}}, \\
& z_{12 n+2}=\frac{(-1)^{n+1} b(1+b e h m)^{2 n+1}(1+3 b e h m)^{n}}{(1+2 b e h m)^{2 n+1}}, \\
& z_{12 n+4}=-\frac{d(1+a d g l)^{2 n+1}(-1+a d g l)^{n}}{(1+2 a d g l)^{n}}, \\
& z_{12 n+6}=\frac{f(-1+c f k o)^{n+1}(1+c f k o)^{2 n+1}}{(1+2 c f k o)^{n}}, \\
& z_{12 n+8}=\frac{(-1)^{n+1} h(1+b e h m)^{2 n+1}(1+3 b e h m)^{n+1}}{(1+2 b e h m)^{2 n+2}},
\end{aligned}
$$

Proof. For $n=0$, the result holds. Now suppose that $n>0$ and that our assumption holds for $n-1$. We have

$$
\begin{aligned}
x_{12 n-7} & =-\frac{k(1+2 c f k o)^{n}}{(-1+c f k o)^{n}(1+c f k o)^{2 n-1}} \\
x_{12 n-5} & =\frac{(-1)^{n-1} m(1+2 b e h m)^{2 n-1}}{(1+b e h m)^{2 n-1}(1+3 b e h m)^{n}} \\
y_{12 n-7} & =\frac{a(1+2 a d g l)^{n-1}}{(-1+a d g l)^{n}(1+a d g l)^{2 n-1}} \\
y_{12 n-5} & =\frac{c(1+2 c f k o)^{n-1}}{(-1+c f k o)^{n}(1+c f k o)^{2 n-1}} \\
z_{12 n-7} & =\frac{(-1)^{n} e(1+2 b e h m)^{2 n-1}}{(1+b e h m)^{2 n}(1+3 b e h m)^{n-1}} \\
z_{12 n-5} & =-\frac{g(1+2 a d g l)^{n}}{(-1+a d g l)^{n-1}(1+a d g l)^{2 n}}
\end{aligned}
$$

$$
\begin{aligned}
x_{12 n-6} & =-\frac{l(-1+a d g l)^{n}(1+a d g l)^{2 n-1}}{(1+2 a d g l)^{n}}, \\
x_{12 n-4} & =-\frac{o(1+c f k o)^{n}(1+c f k o)^{2 n-1}}{(1+2 c f k o)^{n}}, \\
y_{12 n-6} & =\frac{(-1)^{n} b(1+b e h m)^{2 n}(1+3 b e h m)^{n-1}}{(1+2 b e h m)^{2 n-1}}, \\
y_{12 n-4} & =-\frac{d(-1+a d g l)^{n-1}(1+a d g l)^{2 n}}{(1+2 a d g l)^{n}}, \\
z_{12 n-6} & =\frac{f(-1+c f k o)^{n}(1+c f k o)^{2 n-1}}{(1+2 c f k o)^{n-1}}, \\
z_{12 n-4} & =\frac{(-1)^{n} h(1+b e h m)^{2 n-1}(1+3 b e h m)^{n}}{(1+2 b e h m)^{2 n}} .
\end{aligned}
$$

Now, it follows from Eq. (3.1) that

$$
\begin{aligned}
x_{12 n} & =\frac{y_{12 n-4}}{1+y_{12 n-4} x_{12 n-3} z_{12 n-2} y_{12 n-1}} \\
& =\frac{\left(\frac{-d(-1+a d g l)^{n-1}(1+a d g l)^{2 n}}{(1+2 a d g l)^{n}}\right)}{\left[1+\left(\begin{array}{c}
\left(\frac{-d(-1+a d g l)^{n-1}(1+a d g l)^{2 n}}{(1+2 a d g l)^{n}}\right)\left(\frac{a(1+2 a d g l)^{n}}{(-1+a d g l)^{n}(1+a d g l)^{2 n}}\right) \\
\left(\frac{l(-1+a d g l)^{n}(1+a d g l)^{2 n}}{(1+2 a d g l)^{n}}\right)\left(\frac{g(1+2 a d g l)^{n}}{(-1+a d g l)^{n}(1+a d g l)^{2 n}}\right)
\end{array}\right)\right]}
\end{aligned}
$$




$$
\begin{aligned}
& =\frac{\left(\frac{-d(-1+a d g l)^{n-1}(1+a d g l)^{2 n}}{(1+2 a d g l)^{n}}\right)}{\left[1+\left(\frac{-a d g l}{(-1+a d g l)}\right)\right]}=\frac{\left(\frac{-d(-1+a d g l)^{n-1}(1+a d g l)^{2 n}}{(1+2 a d g l)^{n}}\right)}{\left(\frac{-1+a d g l-a d g l}{-1+a d g l}\right)} \\
& =\frac{d(-1+a d g l)^{n}(1+a d g l)^{2 n}}{(1+2 a d g l)^{n}}, \\
& y_{12 n+1}=\frac{z_{12 n-3}}{1-z_{12 n-3} y_{12 n-2} x_{12 n-1} z_{12 n}} \\
& =\frac{\left(\frac{k(1+2 c f k o)^{n}}{(-1+c f k o)^{n}(1+c f k o)^{2 n}}\right)}{\left[1-\left(\begin{array}{c}
\left(\frac{k(1+2 c f k o)^{n}}{(-1+c f k o)^{n}(1+c f k o)^{2 n}}\right)\left(\frac{f(-1+c f k o)^{n}(1+c f k o)^{2 n}}{(1+2 c f k o)^{n}}\right) \\
\left(\frac{c(1+2 c f k o)^{n}}{(-1+c f k o)^{n}(1+c f k o)^{2 n}}\right)\left(\frac{o(-1+c f k o)^{n}(1+c f k o)^{2 n}}{(1+2 c f k o)^{n}}\right)
\end{array}\right)\right]} \\
& =\frac{\left(\frac{(-1)^{n} k(1+2 c f k o)^{n}}{(1-c f k o)^{n}(1+c f k o)^{2 n}}\right)}{(1-c f k o)}=\frac{(-1)^{n} k(1+2 c f k o)^{n}}{(1-c f k o)^{n+1}(1+c f k o)^{2 n}} \text {, } \\
& z_{12 n+1}=\frac{x_{12 n-3}}{-1-x_{12 n-3} z_{12 n-2} y_{12 n-1} x_{12 n}} \\
& =\frac{\left(\frac{a(1+2 a d g l)^{n}}{(-1+a d g l)^{n}(1+a d g l)^{2 n}}\right)}{\left.-1-\left(\begin{array}{c}
\left(\frac{a(1+2 a d g l)^{n}}{(-1+a d g l)^{n}(1+a d g l)^{2 n}}\right)\left(\frac{l(-1+a d g l)^{n}(1+a d g l)^{2 n}}{(1+2 a d g l)^{n}}\right) \\
\left(\frac{g(1+2 a d g l)^{n}}{(-1+a d g l)^{n}(1+a d g l)^{2 n}}\right)\left(\frac{d(-1+a d g l)^{n}(1+a d g l)^{2 n}}{(1+2 a d g l)^{n}}\right)
\end{array}\right)\right]} \\
& =-\frac{\left(\frac{a(1+2 a d g l)^{n}}{(-1+a d g l)^{n}(1+a d g l)^{2 n}}\right)}{(1+a d g l)}=-\frac{a(1+2 a d g l)^{n}}{(-1+a d g l)^{n}(1+a d g l)^{2 n+1}} .
\end{aligned}
$$

Also, we can see that

$$
\begin{aligned}
& x_{12 n+1}=\frac{y_{12 n-3}}{1+y_{12 n-3} x_{12 n-2} z_{12 n-1} y_{12 n}} \\
& =\frac{\left(\frac{(-1)^{n} e(1+2 b e h m)^{2 n}}{(1+b e h m)^{2 n}(1+3 b e h m)^{n}}\right)}{\left.\left[\begin{array}{c}
\left(\frac{(-1)^{n} e(1+2 b e h m)^{2 n}}{(1+b e h m)^{2 n}(1+3 b e h m)^{n}}\right)\left(\frac{(-1)^{n} b(1+b e h m)^{2 n}(1+3 b e h m)^{n}}{(1+2 b e h m)^{2 n}}\right) \\
\left(\frac{(-1)^{n} m(1+2 b h m)^{2 n}}{(1+b h m)^{2 n}(1+3 b h m)^{n}}\right)\left(\frac{(-1)^{n} h(1+b e h m)^{2 n}(1+3 b e h m)^{n}}{(1+2 b e h m)^{2 n}}\right)
\end{array}\right)\right]} \\
& =\frac{\left(\frac{(-1)^{n} e(1+2 b e h m)^{2 n}}{(1+b e h m)^{2 n}(1+3 b e h m)^{n}}\right)}{(1+b e h m)}=\frac{(-1)^{n} e(1+2 b e h m)^{2 n}}{(1+b e h m)^{2 n+1}(1+3 b e h m)^{n}} \text {, } \\
& y_{12 n+5}=\frac{z_{12 n+1}}{1-z_{12 n+4} x_{12 n+3} y_{12 n+2} z_{12 n+1}} \\
& \left.\left.=\frac{\left(-\frac{a(1+2 a d g l)^{n}}{(-1+a d g l)^{n}(1+a d g l)^{2 n+1}}\right)}{\left[1-\left(\left(-\frac{d(1+a d g l)^{2 n+1}(-1+a d g l)^{n}}{(1+2 a d g l)^{n}}\right)\left(\frac{g(1+2 a d g l)^{n+1}}{(-1+a d g l)^{n}(1+a d g l)^{2 n+1}}\right)\right.\right.}\right)\right] \\
& =\frac{\left(-\frac{a(1+2 a d g l)^{n}}{(-1+a d g l)^{n}(1+a d g l)^{2 n+1}}\right)}{(1-a d g l)}=\frac{a(1+2 a d g l)^{n}}{(-1+a d g l)^{n+1}(1+a d g l)^{2 n+1}} \\
& =\frac{a(1+2 a d g l)^{n}}{(-1+a d g l)^{2 n}(1+a d g l)^{2 n+1}}, \\
& z_{12 n+6}=\frac{x_{12 n+2}}{-1-x_{12 n+5} y_{12 n+4} z_{12 n+3} x_{12 n+2}} \\
& =\frac{\left(-\frac{f(-1+c f k o)^{n+1}(1+c f k o)^{2 n}}{(1+2 c f k o)^{n}}\right)}{-1-\left[\left(-\frac{k(1+2 c f k o)^{n+1}}{(-1+c f k o)^{n+1}(1+c f k o)^{2 n+1}}\right)\left(\frac{o(-1+c f k o)^{n}(1+c f k o)^{2 n+1}}{(1+2 c f k o)^{n+1}}\right)\right.}
\end{aligned}
$$




$$
\begin{aligned}
& =\frac{\left(-\frac{f(-1+c f k o)^{n+1}(1+c f k o)^{2 n}}{(1+2 c f k o)^{n}}\right)}{-1+\left(\frac{c f k o}{1+c f k o}\right)}=\frac{\left(-\frac{f(-1+c f k o)^{n+1}(1+c f k o)^{2 n}}{(1+2 c f k o)^{n}}\right)}{\frac{-1-c f k o+c f k o}{1+c f k o}} \\
& =\frac{f(-1+c f k o)^{n+1}(1+c f k o)^{2 n+1}}{(1+2 c f k o)^{n}} .
\end{aligned}
$$

Also, we can prove the other relations. This completes the proof.

Theorem 3.2. Assume that $\left\{x_{n}, y_{n}, z_{n}\right\}$ are solutions of the system

$$
x_{n+1}=\frac{y_{n-3}}{-1+y_{n-3} z_{n-1} x_{n-2} y_{n}}, y_{n+1}=\frac{z_{n-3}}{1-z_{n-3} x_{n-1} y_{n-2} z_{n}}, z_{n+1}=\frac{x_{n-3}}{1-x_{n-3} y_{n-1} z_{n-2} x_{n}},
$$

then for $n=0,1,2, \ldots$, we see that

$$
\begin{aligned}
& x_{12 n-3}=\frac{a(1-2 a d g l)^{2 n}}{(-1+a d g l)^{2 n}(-1+3 a d g l)^{n}}, \\
& x_{12 n-1}=\frac{c(-1+2 c f k o)^{n}}{(-1+c f k o)^{2 n}(1+c f k o)^{n}} \text {, } \\
& x_{12 n+1}=\frac{e(1+2 b e h m)^{n}}{(-1+b e h m)^{n+1}(1+b e h m)^{2 n}}, \\
& x_{12 n+3}=-\frac{g(-1+2 a d g l)^{2 n+1}}{(-1+3 a d g l)^{n+1}(-1+a d g l)^{2 n}}, \\
& x_{12 n+5}=\frac{k(-1+2 c f k o)^{n}}{(-1+c f k o)^{n+1}(1+c f k o)^{2 n+1}}, \\
& x_{12 n+7}=\frac{m(1+2 b e h m)^{n}}{(-1+b e h m)^{n+1}(1+b e h m)^{2 n+1}} \text {, } \\
& y_{12 n-3}=\frac{e(1+2 b e h m)^{n}}{(-1+b e h m)^{n}(1+b e h m)^{2 n}}, \\
& y_{12 n-1}=\frac{g(1-2 a d g l)^{2 n}}{(-1+a d g l)^{2 n}(-1+3 a d g l)^{n}}, \\
& y_{12 n+1}=-\frac{k(-1+2 c f k o)^{n}}{(-1+c f k o)^{2 n+1}(1+c f k o)^{n}}, \\
& y_{12 n+3}=\frac{m(1+2 b e m)^{n}}{(-1+b e m)^{n}(1+b e m)^{2 n+1}}, \\
& y_{12 n+5}=-\frac{a(-1+2 a d g l)^{2 n+1}}{(-1+a d g l)^{2 n+2}(-1+3 a d g l)^{n}}, \\
& y_{12 n+7}=-\frac{c(-1+2 c f k o)^{n+1}}{(-1+c f k o)^{2 n}(1+c f k o)^{n}}, \\
& z_{12 n-3}=\frac{k(-1+2 c f k o)^{n}}{(-1+c f k o)^{2 n}(1+c f k o)^{n}}, \\
& z_{12 n-1}=\frac{m(1+2 b e h m)^{n}}{(-1+\text { behm })^{n}(1+\text { behm })^{2 n}}, \\
& z_{12 n+1}=-\frac{a(1-2 a d g l)^{2 n}}{(-1+a d g l)^{2 n+1}(-1+3 a d g l)^{n}}, \\
& z_{12 n+3}=\frac{c(-1+2 c f k o)^{n+1}}{(-1+c f k o)^{2 n+1}(1+c f k o)^{n}}, \\
& z_{12 n+5}=\frac{e(1+2 b e h m)^{n+1}}{(-1+b e h m)^{n+1}(1+b e h m)^{2 n+1}}, \\
& z_{12 n+7}=\frac{g(-1+2 a d g l)^{2 n+1}}{(-1+a d g l)^{2 n+1}(-1+3 a d g l)^{n+1}},
\end{aligned}
$$

$$
\begin{aligned}
& x_{12 n-2}=\frac{b(-1+b e h m)^{n}(1+b e h m)^{2 n}}{(1+2 b e h m)^{n}}, \\
& x_{12 n}=\frac{d(-1+a d g l)^{2 n}(-1+3 a d g l)^{n}}{(1-2 a d g l)^{2 n}}, \\
& x_{12 n+2}=-\frac{f(-1+c f k o)^{2 n+1}(1+c f k o)^{n}}{(-1+2 c f k o)^{n+1}} \text {, } \\
& x_{12 n+4}=-\frac{h(1+b e h m)^{2 n+1}(-1+b e h m)^{n}}{(1+2 b e h m)^{n+1}} \text {, } \\
& x_{12 n+6}=\frac{l(-1+a d g l)^{2 n+2}(-1+3 a d g l)^{n}}{(-1+2 a d g l)^{2 n+1}} \text {, } \\
& x_{12 n+8}=\frac{o(-1+c f k o)^{2 n+2}(1+c f k o)^{n}}{(-1+2 c f k o)^{n+1}}, \\
& y_{12 n-2}=\frac{f(-1+c f k o)^{2 n}(1+c f k o)^{n}}{(-1+2 c f k o)^{n}}, \\
& y_{12 n}=\frac{h(-1+b e h m)^{n}(1+b e h m)^{2 n}}{(1+2 b e h m)^{n}}, \\
& y_{12 n+2}=\frac{l(-1+a d g l)^{2 n+1}(-1+3 a d g l)^{n}}{(-1+2 a d g l)^{2 n+1}} \text {, } \\
& y_{12 n+4}=-\frac{o(-1+c f k o)^{2 n+1}(1+c f k o)^{n}}{(-1+2 c f k o)^{n}} \text {, } \\
& y_{12 n+6}=-\frac{b(-1+b e h m)^{n+1}(1+b e h m)^{2 n+1}}{(1+2 b e h m)^{n}}, \\
& y_{12 n+8}=\frac{d(-1+a d g l)^{n+1}(-1+3 a d g l)^{2 n+1}}{(1-2 a d g l)^{2 n+2}}, \\
& z_{12 n-2}=\frac{l(-1+a d g l)^{2 n}(-1+3 a d g l)^{n}}{(1-2 a d g l)^{2 n}}, \\
& z_{12 n}=\frac{o(-1+c f k o)^{2 n}(1+c f k o)^{n}}{(-1+2 c f k o)^{n}}, \\
& z_{12 n+2}=-\frac{b(-1+b e h m)^{n+1}(1+b e h m)^{2 n}}{(1+2 b e h m)^{n}}, \\
& z_{12 n+4}=\frac{d(-1+3 a d g l)^{n+1}(-1+a d g l)^{2 n}}{(-1+2 a d g l)^{2 n+1}}, \\
& z_{12 n+6}=-\frac{f(-1+c f k o)^{2 n+1}(1+c f k o)^{n+1}}{(-1+2 c f k o)^{n+1}}, \\
& z_{12 n+8}=\frac{h(-1+b e h m)^{n+1}(1+b e h m)^{2 n+1}}{(1+2 b e h m)^{n+1}} .
\end{aligned}
$$

Theorem 3.3. Let $\left\{x_{n}, y_{n}, z_{n}\right\}$ be solutions of the system

$$
x_{n+1}=\frac{y_{n-3}}{-1+y_{n-3} z_{n-1} x_{n-2} y_{n}}, y_{n+1}=\frac{z_{n-3}}{-1-z_{n-3} x_{n-1} y_{n-2} z_{n}}, z_{n+1}=\frac{x_{n-3}}{-1-x_{n-3} y_{n-1} z_{n-2} x_{n}},
$$

then we get

$$
x_{12 n-3}=\frac{a(-1+2 a d g l)^{n}}{(-1+a d g l)^{2 n}(1+a d g l)^{n}}, \quad x_{12 n-2}=\frac{b(-1+b e h m)^{2 n}(1+b e h m)^{n}}{(-1+2 b e h m)^{n}}
$$




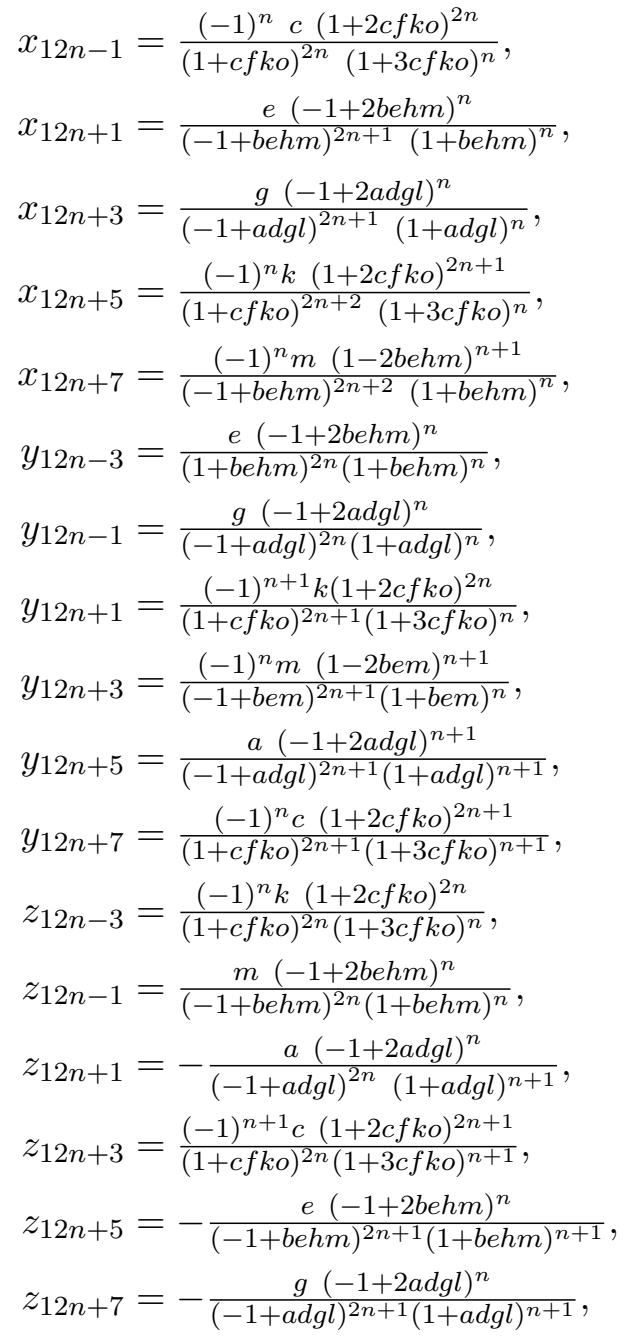

$$
\begin{aligned}
& x_{12 n}=\frac{d(-1+a d g l)^{2 n}(1+a d g l)^{n}}{(-1+2 a d g l)^{n}}, \\
& x_{12 n+2}=\frac{(-1)^{n+1} f(1+c f k o)^{2 n+1}(1+3 c f k o)^{n}}{(1+2 c f k o)^{2 n+1}}, \\
& x_{12 n+4}=\frac{h(-1+b e h m)^{2 n+1}(1+b e h m)^{n}}{(-1+2 b e h m)^{n}} \text {, } \\
& x_{12 n+6}=-\frac{l(-1+a d g l)^{2 n+1}(1+a d g l)^{n+1}}{(-1+2 a d g l)^{n}}, \\
& x_{12 n+8}=\frac{(-1)^{n} o(1+c f k o)^{2 n+1}(1+3 c f k o)^{n+1}}{(1+2 c f k o)^{2 n+2}} \text {, } \\
& y_{12 n-2}=\frac{(-1)^{n} f(1+c f k o)^{2 n}(1+3 c f k o)^{n}}{(1+2 c f k o)^{2 n}}, \\
& y_{12 n}=\frac{h(-1+b e h m)^{2 n}(1+b e h m)^{n}}{(-1+2 b e h m)^{n}}, \\
& y_{12 n+2}=-\frac{l(1+a d g l)^{n+1}(-1+a d g l)^{2 n}}{(-1+2 a d g l)^{n}}, \\
& y_{12 n+4}=\frac{(-1)^{n+1} o(1+c f k o)^{2 n}(1+3 c f k o)^{n+1}}{(1+2 c f k o)^{2 n+1}} \text {, } \\
& y_{12 n+6}=\frac{b(-1+b e h m)^{2 n+1}(1+b e h m)^{n+1}}{(-1+2 b e h m)^{n+1}}, \\
& y_{12 n+8}=\frac{d(-1+a d g l)^{2 n+1}(1+a d g l)^{n+1}}{(-1+2 a d g l)^{n+1}}, \\
& z_{12 n-2}=\frac{l(-1+a d g l)^{2 n}(1+a d g l)^{n}}{(-1+2 a d g l)^{n}}, \\
& z_{12 n}=\frac{(-1)^{n} o(1+c f k o)^{2 n}(1+3 c f k o)^{n}}{(1+2 c f k o)^{2 n}}, \\
& z_{12 n+2}=-\frac{b(-1+b e h m)^{2 n+1}(1+b e h m)^{n}}{(-1+2 b e h m)^{n+1}}, \\
& z_{12 n+4}=-\frac{d(-1+a d g l)^{2 n+1}(1+a d g l)^{n}}{(-1+2 a d g l)^{n+1}}, \\
& z_{12 n+6}=\frac{(-1)^{n} f(1+c f k o)^{2 n+2}(1+3 c f k o)^{n}}{(1+2 c f k o)^{2 n+1}}, \\
& z_{12 n+8}=-\frac{h(-1+b e h m)^{2 n+2}(1+b e h m)^{n}}{(-1+2 b e h m)^{n+1}} .
\end{aligned}
$$

Theorem 3.4. Let $\left\{x_{n}, y_{n}, z_{n}\right\}$ be solutions of the system

$$
x_{n+1}=\frac{y_{n-3}}{-1+y_{n-3} z_{n-1} x_{n-2} y_{n}}, y_{n+1}=\frac{z_{n-3}}{1-z_{n-3} x_{n-1} y_{n-2} z_{n}}, z_{n+1}=\frac{x_{n-3}}{-1-x_{n-3} y_{n-1} z_{n-2} x_{n}},
$$

then we see that

$$
\begin{aligned}
x_{12 n-3} & =a \prod_{i=0}^{n-1} \frac{(1+12 i a d g l)(1+(12 i+4) a d g l)(1+(12 i+8) a d g l)}{(1+(12 i+1) a d g l)(1+(12 i+5) a d g l)(1+(12 i+9) a d g l)}, \\
x_{12 n-2} & =b \prod_{i=0}^{n-1} \frac{(-1+(12 i+1) b e h m)(-1+(12 i+5) b e h m)(-1+(12 i+9) b e h m)}{(-1+(12 i+2) b e h m)(-1+(12 i+6) b e h m)(-1+(12 i+10) b e h m)}, \\
x_{12 n-1} & =c \prod_{i=0}^{n-1} \frac{(-1+(12 i+2) c f k o)(-1+(12 i+6) c f k o)(-1+(12 i+10) c f k o)}{(-1+(12 i+3) c f k o)(-1+(12 i+7) c f k o)(-1+(12 i+11) c f k o)}, \\
x_{12 n} & =d \prod_{i=0}^{n-1} \frac{(1+(12 i+3) a d g l)(1+(12 i+7) a d g l)(1+(12 i+11) a d g l)}{(1+(12 i+4) a d g l)(1+(12 i+8) a d g l)(1+(12 i+12) a d g l)}, \\
x_{12 n+1} & =\frac{e}{(-1+b e h m)} \prod_{i=0}^{n-1} \frac{(-1+(12 i+4) b e h m)(-1+(12 i+8) b e h m)(-1+(12 i+12) b e h m)}{(-1+(12 i+5) b e h m)(-1+(12 i+9) b e h m)(-1+(12 i+13) b e h m)} \\
x_{12 n+2} & =-\frac{f(-1+c f k o)}{(-1+2 c f k o)} \prod_{i=0}^{n-1} \frac{(-1+(12 i+5) c f k o)(-1+(12 i+9) c f k o)(-1+(12 i+13) c f k o)}{(-1+(12 i+6) c f k o)(-1+(12 i+10) c f k o)(-1+(12 i+14) c f k o)}, \\
x_{12 n+3} & =-\frac{g(1+2 a d g l)}{(1+3 a d g l)} \prod_{i=0}^{n-1} \frac{(1+(12 i+6) a d g l)(1+(12 i+10) a d g l)(1+(12 i+14) a d g l)}{(1+(12 i+7) a d g l)(1+(12 i+11) a d g l)(1+(12 i+15) a d g l)},
\end{aligned}
$$




$$
\begin{aligned}
& x_{12 n+4}=-\frac{h(-1+3 b e h m)}{(-1+4 b e h m)} \prod_{i=0}^{n-1} \frac{(-1+(12 i+7) \text { behm })(-1+(12 i+11) \text { behm })(-1+(12 i+15) \text { behm })}{(-1+(12 i+8) \text { behm })(-1+(12 i+12) \text { behm })(-1+(12 i+16) \text { behm })}, \\
& x_{12 n+5}=\frac{k(-1+4 c f k o)}{(-1+c f k o)(-1+5 c f k o)} \prod_{i=0}^{n-1} \frac{(-1+(12 i+8) c f k o)(-1+(12 i+12) c f k o)(-1+(12 i+16) c f k o)}{(-1+(12 i+9) c f k o)(-1+(12 i+13) c f k o)(-1+(12 i+17) c f k o)}, \\
& x_{12 n+6}=-\frac{l(1+a d g l)(1+5 a d g l)}{(1+2 a d g l)(1+6 a d g l)} \prod_{i=0}^{n-1} \frac{(1+(12 i+9) a d g l)(1+(12 i+13) a d g l)(1+(12 i+17) a d g l)}{(1+(12 i+10) a d g l)(1+(12 i+14) a d g l)(1+(12 i+18) a d g l)}, \\
& x_{12 n+7}=-\frac{m(-1+2 b e h m)(-1+6 b e h m)}{(-1+3 b e h m)(-1+7 b e h m)} \prod_{i=0}^{n-1} \frac{(-1+(12 i+10) \text { behm })(-1+(12 i+14) \text { behm })(-1+(12 i+18) \text { behm })}{(-1+(12 i+11) \text { behm })(-1+(12 i+15) \text { behm })(-1+(12 i+19) \text { behm })}, \\
& x_{12 n+8}=-\frac{o(-1+3 c f k o)(-1+7 c f k o)}{(-1+4 c f k o)(-1+8 c f k o)} \prod_{i=0}^{n-1} \frac{(-1+(12 i+11) c f k o)(-1+(12 i+15) c f k o)(-1+(12 i+19) c f k o)}{(-1+(12 i+12) c f k o)(-1+(12 i+16) c f k o)(-1+(12 i+20) c f k o)}, \\
& y_{12 n-3}=-e \prod_{i=0}^{n-1} \frac{(-1+(12 i) \text { behm })(-1+(12 i+4) \text { behm })(-1+(12 i+8) \text { behm })}{(-1+(12 i+1) \text { behm })(-1+(12 i+5) \text { behm })(-1+(12 i+9) \text { behm })}, \\
& y_{12 n-2}=f \prod_{i=0}^{n-1} \frac{(-1+(12 i+1) c f k o)(-1+(12 i+5) c f k o)(-1+(12 i+9) c f k o)}{(-1+(12 i+2) c f k o)(-1+(12 i+6) c f k o)(-1+(12 i+10) c f k o)}, \\
& y_{12 n-1}=g \prod_{i=0}^{n-1} \frac{(1+(12 i+2) a d g l)(1+(12 i+6) a d g l)(1+(12 i+10) a d g l)}{(1+(12 i+3) a d g l)(1+(12 i+7) a d g l)(1+(12 i+11) a d g l)}, \\
& y_{12 n}=h \prod_{i=0}^{n-1} \frac{(-1+(12 i+3) \text { behm })(-1+(12 i+7) \text { behm })(-1+(12 i+11) \text { behm })}{(-1+(12 i+4) \text { behm })(-1+(12 i+8) \text { behm })(-1+(12 i+12) \text { behm })}, \\
& y_{12 n+1}=-\frac{k}{(-1+c f k o)} \prod_{i=0}^{n-1} \frac{(-1+(12 i+4) c f k o)(-1+(12 i+8) c f k o)(-1+(12 i+12) c f k o)}{(-1+(12 i+5) c f k o)(-1+(12 i+9) c f k o)(-1+(12 i+13) c f k o)} \text {, } \\
& y_{12 n+2}=\frac{l(1+a d g l)}{(1+2 a d g l)} \prod_{i=0}^{n-1} \frac{(1+(12 i+5) a d g l)(1+(12 i+9) a d g l)(1+(12 i+13) a d g l)}{(1+(12 i+6) a d g l)(1+(12 i+10) a d g l)(1+(12 i+14) a d g l)}, \\
& y_{12 n+3}=\frac{m(-1+2 b e h m)}{(-1+3 b e h m)} \prod_{i=0}^{n-1} \frac{(-1+(12 i+6) \text { behm })(-1+(12 i+10) \text { behm })(-1+(12 i+14) \text { behm })}{(-1+(12 i+7) \text { behm })(-1+(12 i+11) \text { behm })(-1+(12 i+15) \text { behm })}, \\
& y_{12 n+4}=\frac{o(-1+3 c f k o)}{(-1+4 c f k o)} \prod_{i=0}^{n-1} \frac{(-1+(12 i+7) c f k o)(-1+(12 i+11) c f k o)(-1+(12 i+15) c f k o)}{(-1+(12 i+8) c f k o)(-1+(12 i+12) c f k o)(-1+(12 i+16) c f k o)}, \\
& y_{12 n+5}=-\frac{a(1+4 a d g l)}{(1+a d g l)(1+5 a d g l)} \prod_{i=0}^{n-1} \frac{(1+(12 i+8) a d g l)(1+(12 i+12) a d g l)(1+(12 i+16) a d g l)}{(1+(12 i+9) a d g l)(1+(12 i+13) a d g l)(1+(12 i+17) a d g l)}, \\
& y_{12 n+6}=-\frac{b(-1+\text { behm })(-1+5 b e h m)}{(-1+2 b e h m)(-1+6 b e h m)} \prod_{i=0}^{n-1} \frac{(-1+(12 i+9) \text { behm })(-1+(12 i+13) \text { behm })(-1+(12 i+17) \text { behm })}{(-1+(12 i+10) \text { behm })(-1+(12 i+14) \text { behm })(-1+(12 i+18) \text { behm })}, \\
& y_{12 n+7}=-\frac{c(-1+2 c f k o)(-1+6 c f k o)}{(-1+3 c f k o)(-1+7 c f k o)} \prod_{i=0}^{n-1} \frac{(-1+(12 i+10) c f k o)(-1+(12 i+14) c f k o)(-1+(12 i+18) c f k o)}{(-1+(12 i+11) c f k o)(-1+(12 i+15) c f k o)(-1+(12 i+19) c f k o)}, \\
& y_{12 n+8}=-\frac{d(1+3 a d g l)(1+7 a d g l)}{(1+4 a d g l)(1+8 a d g l)} \prod_{i=0}^{n-1} \frac{(1+(12 i+11) a d g l)(1+(12 i+15) a d g l)(1+(12 i+19) a d g l)}{(1+(12 i+12) a d g l)(1+(12 i+16) a d g l)(1+(12 i+20) a d g l)}, \\
& z_{12 n-3}=-k \prod_{i=0}^{n-1} \frac{(-1+(12 i) c f k o)(-1+(12 i+4) c f k o)(-1+(12 i+8) c f k o)}{(-1+(12 i+1) c f k o)(-1+(12 i+5) c f k o)(-1+(12 i+9) c f k o)}, \\
& z_{12 n-2}=l \prod_{i=0}^{n-1} \frac{(1+(12 i+1) a d g l)(1+(12 i+5) a d g l)(1+(12 i+9) a d g l)}{(1+(12 i+2) a d g l)(1+(12 i+6) a d g l)(1+(12 i+10) a d g l)}, \\
& z_{12 n-1}=m \prod_{i=0}^{n-1} \frac{(-1+(12 i+2) \text { behm })(-1+(12 i+6) \text { behm })(-1+(12 i+10) \text { behm })}{(-1+(12 i+3) \text { behm })(-1+(12 i+7) \text { behm })(-1+(12 i+11) \text { behm })}, \\
& z_{12 n}=o \prod_{i=0}^{n-1} \frac{(-1+(12 i+3) c f k o)(-1+(12 i+7) c f k o)(-1+(12 i+11) c f k o)}{(-1+(12 i+4) c f k o)(-1+(12 i+8) c f k o)(-1+(12 i+12) c f k o)},
\end{aligned}
$$




$$
\begin{aligned}
& z_{12 n+1}=-\frac{a}{(1+a d g l)} \prod_{i=0}^{n-1} \frac{(1+(12 i+4) a d g l)(1+(12 i+8) a d g l)(1+(12 i+12) a d g l)}{(1+(12 i+5) a d g l)(1+(12 i+9) a d g l)(1+(12 i+13) a d g l)}, \\
& z_{12 n+2}=-\frac{b(-1+\text { behm })}{(-1+2 b e h m)} \prod_{i=0}^{n-1} \frac{(-1+(12 i+5) \text { behm })(-1+(12 i+9) \text { behm })(-1+(12 i+13) \text { behm })}{(-1+(12 i+6) \text { behm })(-1+(12 i+10) \text { behm })(-1+(12 i+14) \text { behm })}, \\
& z_{12 n+3}=-\frac{c(-1+2 c f k o)}{(-1+3 c f k o)} \prod_{i=0}^{n-1} \frac{(-1+(12 i+6) c f k o)(-1+(12 i+10) c f k o)(-1+(12 i+14) c f k o)}{(-1+(12 i+7) c f k o)(-1+(12 i+11) c f k o)(-1+(12 i+15) c f k o)}, \\
& z_{12 n+4}=-\frac{d(1+3 a d g l)}{(1+4 a d g l)} \prod_{i=0}^{n-1} \frac{(1+(12 i+7) a d g l)(1+(12 i+11) a d g l)(1+(12 i+15) a d g l)}{(1+(12 i+8) a d g l)(1+(12 i+12) a d g l)(1+(12 i+16) a d g l)} \\
& z_{12 n+5}=-\frac{e(-1+4 b e h m)}{(-1+\text { behm })(-1+5 b e h m)} \prod_{i=0}^{n-1} \frac{(-1+(12 i+8) \text { behm })(-1+(12 i+12) \text { behm })(-1+(12 i+16) \text { behm })}{(-1+(12 i+9) \text { behm })(-1+(12 i+13) \text { behm })(-1+(12 i+17) \text { behm })}, \\
& z_{12 n+6}=\frac{f(-1+c f k o)(-1+5 c f k o)}{(-1+2 c f k o)(-1+6 c f k o)} \prod_{i=0}^{n-1} \frac{(-1+(12 i+9) c f k o)(-1+(12 i+13) c f k o)(-1+(12 i+17) c f k o)}{(-1+(12 i+10) c f k o)(-1+(12 i+14) c f k o)(-1+(12 i+18) c f k o)}, \\
& z_{12 n+7}=\frac{g(1+2 a d g l)(1+6 a d g l)}{(1+3 a d g l)(1+7 a d g l)} \prod_{i=0}^{n-1} \frac{(1+(12 i+10) a d g l)(1+(12 i+14) a d g l)(1+(12 i+18) a d g l)}{(1+(12 i+11) a d g l)(1+(12 i+15) a d g l)(1+(12 i+19) a d g l)}, \\
& z_{12 n+8}=\frac{h(-1+3 b e h m)(-1+7 b e h m)}{(-1+4 b e h m)(-1+8 b e h m)} \prod_{i=0}^{n-1} \frac{(-1+(12 i+11) \text { behm })(-1+(12 i+15) \text { behm })(-1+(12 i+19) \text { behm })}{(-1+(12 i+12) \text { behm })(-1+(12 i+16) \text { behm })(-1+(12 i+20) \text { behm })}
\end{aligned}
$$

where $\prod_{i=0}^{-1} A_{i}=1$.

4. On the system: $x_{n+1}=\frac{y_{n-3}}{ \pm 1+y_{n} z_{n-1} x_{n-2} y_{n-3}}, y_{n+1}=\frac{z_{n-3}}{ \pm 1+z_{n} x_{n-1} y_{n-2} z_{n-3}}, z_{n+1}=\frac{x_{n-3}}{ \pm 1+x_{n} y_{n-1} z_{n-2} x_{n-3}}$

In this section, we study the solutions and periodicity of the following system

$$
x_{n+1}=\frac{y_{n-3}}{-1+y_{n} z_{n-1} x_{n-2} y_{n-3}}, y_{n+1}=\frac{z_{n-3}}{-1+z_{n} x_{n-1} y_{n-2} z_{n-3}}, z_{n+1}=\frac{x_{n-3}}{-1+x_{n} y_{n-1} z_{n-2} x_{n-3}},
$$

with nonzero real initial conditions.

Theorem 4.1. The solutions of system (4.1) are given by the following formulas

$$
\begin{aligned}
x_{12 n-3} & =\frac{a}{(-1+a d g l)^{3 n}}, \\
x_{12 n} & =d(-1+a d g l)^{3 n}, \\
x_{12 n+3} & =\frac{g}{(-1+a d g l)^{3 n+1}}, \\
x_{12 n+6} & =l(-1+a d g l)^{3 n+2}, \\
y_{12 n-3} & =\frac{e}{(-1+b e h m)^{3 n+1}}, \\
y_{12 n} & =h(-1+b e h m)^{3 n}, \\
y_{12 n+3} & =\frac{m}{(-1+b e h m)^{3 n+1}}, \\
y_{12 n+6} & =b(-1+b e h m)^{3 n+2}, \\
z_{12 n-3} & =\frac{k}{(-1+c f k o)^{3 n}}, \\
z_{12 n} & =o(-1+c f k o)^{3 n}, \\
z_{12 n+3} & =\frac{c}{(-1+c f k o)^{3 n+1}}, \\
z_{12 n+6} & =f(-1+c f k o)^{3 n+2}, \\
\text { for } n= & 0,1,2, \ldots .
\end{aligned}
$$

$$
\begin{aligned}
x_{12 n-2} & =b(-1+\text { behm })^{3 n}, & x_{12 n-1} & =\frac{c}{(-1+c f k o)^{3 n}}, \\
x_{12 n+1} & =\frac{e}{(-1+b e h m)^{3 n+1}}, & x_{12 n+2} & =f(-1+c f k o)^{3 n+1}, \\
x_{12 n+4} & =h(-1+b e h m)^{3 n+1}, & x_{12 n+5} & =\frac{k}{(-1+c f k o)^{3 n+2}}, \\
x_{12 n+7} & =\frac{m}{(-1+b e h m)^{3 n+2}}, & x_{12 n+8} & =o(-1+c f k o)^{3 n+2}, \\
y_{12 n-2} & =f(-1+c f k o)^{3 n+1}, & y_{12 n-1} & =\frac{g}{(-1+a d g l)^{3 n}}, \\
y_{12 n+1} & =\frac{k}{(-1+c f k o)^{3 n+1}}, & y_{12 n+2} & =l(-1+a d g l)^{3 n+1}, \\
y_{12 n+4} & =o(-1+c f k o)^{3 n+1}, & y_{12 n+5} & =\frac{a}{(-1+a d g l)^{3 n+2}}, \\
y_{12 n+7} & =\frac{c}{(-1+c f k o)^{3 n+2}}, & y_{12 n+8} & =d(-1+a d g l)^{3 n+2}, \\
z_{12 n-2} & =(-1+a d g l)^{3 n}, & z_{12 n-1} & =\frac{m}{(-1+b e h m)^{3 n}}, \\
z_{12 n+1} & =\frac{a}{(-1+a d g l)^{3 n+1}}, & z_{12 n+2} & =b(-1+b e h m)^{3 n+1}, \\
z_{12 n+4} & =d(-1+a d g l)^{3 n+1}, & z_{12 n+5} & =\frac{e}{(-1+b e h m)^{3 n+2}}, \\
z_{12 n+7} & =\frac{g}{(-1+a d g l)^{3 n+2}}, & z_{12 n+8} & =h(-1+b e h m)^{3 n+2},
\end{aligned}
$$


Lemma 4.2. The solutions of system (4.1) are unbounded except in the following two theorems.

Theorem 4.3. System (4.1) has a periodic solution of period twelve, iff adgl $=b e h m=c f k o=-2$ and it will take the following form

$$
\begin{aligned}
& \left\{x_{n}\right\}=\left\{x_{-3}, x_{-2}, x_{-1}, x_{0}, z_{-3}, z_{-2}, z_{-1}, z_{0}, y_{-3}, y_{-2}, y_{-1}, y_{0}, x_{-3}, x_{-2}, x_{-1}, x_{0}, \ldots\right\} \\
& \left\{y_{n}\right\}=\left\{y_{-3}, y_{-2}, y_{-1}, y_{0}, x_{-3}, x_{-2}, x_{-1}, x_{0}, z_{-3}, z_{-2}, z_{-1}, z_{0}, y_{-3}, y_{-2}, y_{-1}, y_{0}, \ldots\right\} \\
& \left\{z_{n}\right\}=\left\{z_{-3}, z_{-2}, z_{-1}, z_{0}, y_{-3}, y_{-2}, y_{-1}, y_{0}, x_{-3}, x_{-2}, x_{-1}, x_{0}, z_{-3}, z_{-2}, z_{-1}, z_{0}, \ldots\right\} .
\end{aligned}
$$

Theorem 4.4. System 4.1) has a periodic solution of period four, iff $x_{-i}=y_{-i}=z_{-i}, i=0,1,2,3$, $a d g l=-2$ and will be in the form $\left\{x_{n}\right\}=\left\{y_{n}\right\}=\left\{z_{n}\right\}=\left\{x_{-3}, x_{-2}, x_{-1}, x_{0}, x_{-3}, x_{-2}, \ldots\right\}$.

\section{Numerical examples}

Here we consider some numerical examples for the previous systems to illustrate the results.

Example 5.1. We consider numerical example for the difference equations system (2.1) with the initial conditions $x_{-3}=0.5, x_{-2}=0.11, x_{-1}=-0.28, x_{0}=1.3, y_{-3}=0.3, y_{-2}=2, y_{-1}=0.2, y_{0}=5, z_{-3}=$ $0.8, z_{-2}=0.4, z_{-1}=-0.1$ and $z_{0}=0.7$, (see Figure 1 ).

Example 5.2. We assume the system of difference equation 2.2 with the initial conditions $x_{-3}=$ $0.5, x_{-2}=0.11, x_{-1}=0.28, x_{0}=-1.3, y_{-3}=0.21, y_{-2}=0.52, y_{-1}=0.2, y_{0}=0.31, z_{-3}=-0.6, z_{-2}=$ $0.4, z_{-1}=-0.1$ and $z_{0}=0.7$, (see Figure 2).

Example 5.3. We consider the difference system (3.1) with the initial conditions $x_{-3}=0.5, x_{-2}=$ $0.11, x_{-1}=-0.28, x_{0}=1.3, y_{-3}=0.21, y_{-2}=2, y_{-1}=0.2, y_{0}=0.75, z_{-3}=0.6, z_{-2}=0.4, z_{-1}=-0.1$ and $z_{0}=0.7$, (see Figure 3).

Example 5.4. Suppose that the initial conditions are $x_{-3}=5, x_{-2}=-6, x_{-1}=0.8, x_{0}=-3, y_{-3}=$ $-7, y_{-2}=4, y_{-1}=0.7, y_{0}=-3, z_{-3}=5, z_{-2}=-20 / 105, z_{-1}=-1 / 63$ and $z_{0}=1 / 8$, for the system (4.1), (see Figure 4).

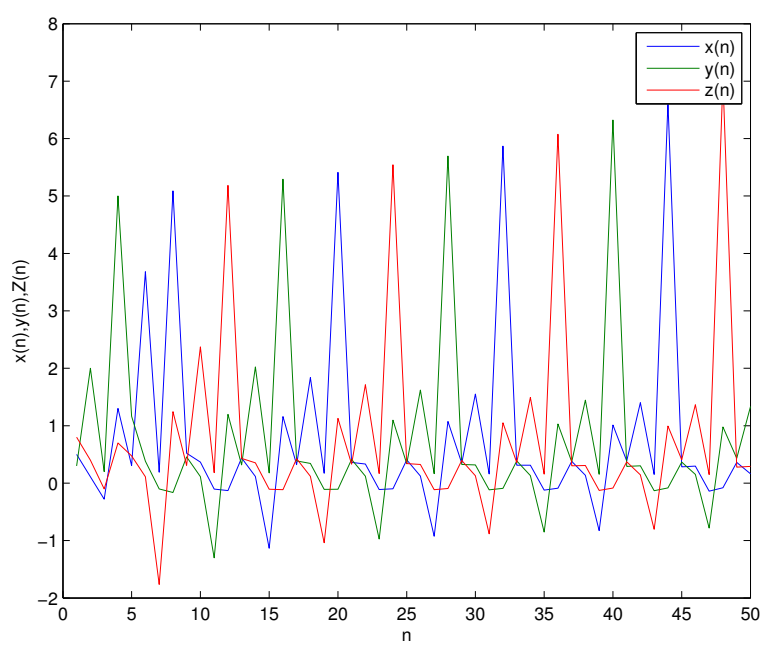

Figure 1: Plot of the system 2.1.

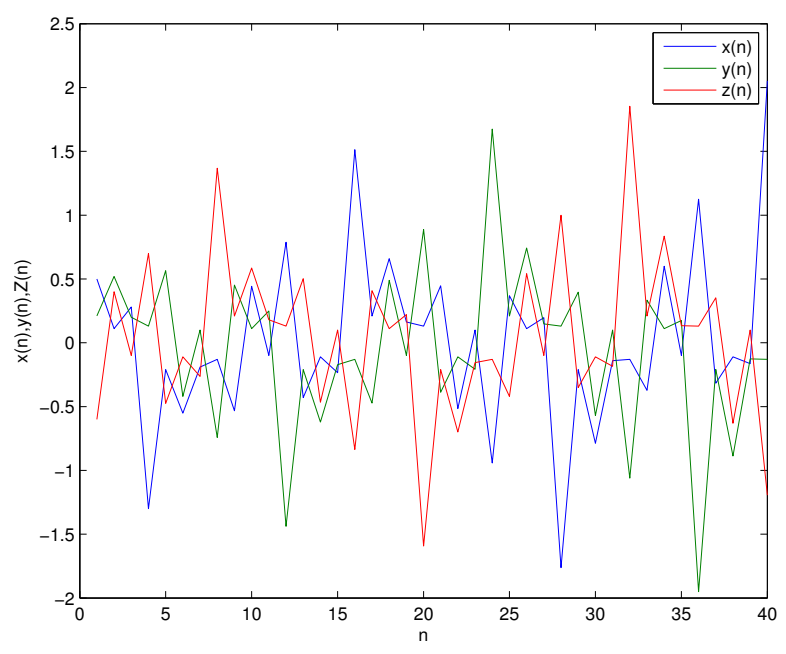

Figure 2: Plot of the system 2.2. 


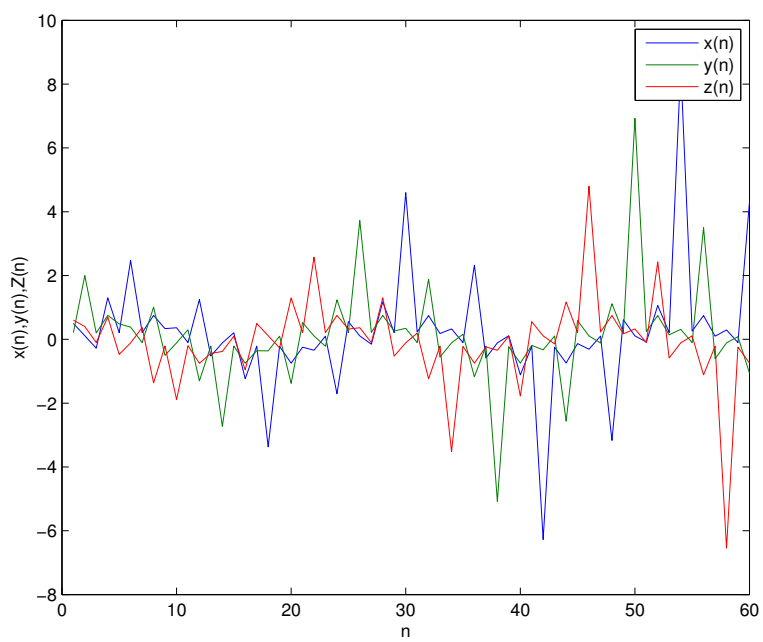

Figure 3: Plot of the system 3.1.

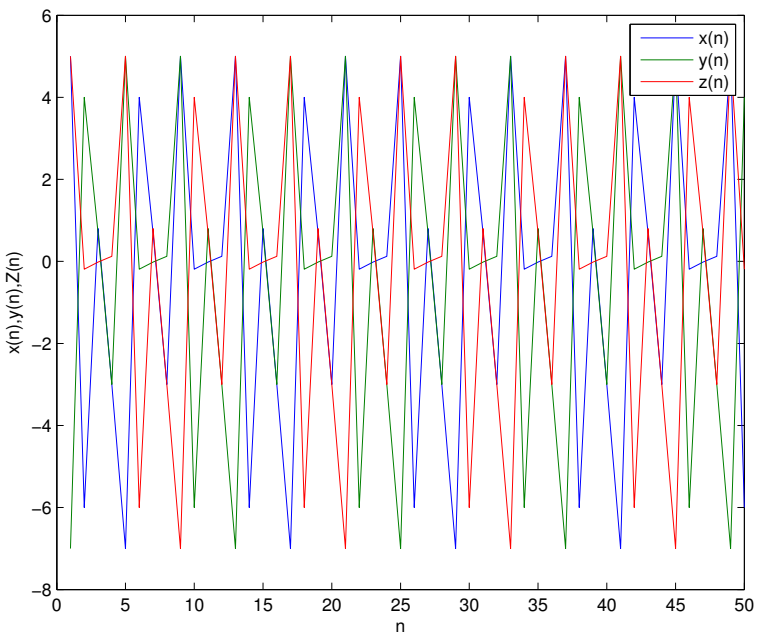

Figure 4: Plot of the system 4.1).

\section{Acknowledgment}

This article was funded by the Deanship of Scientific Research (DSR), King Abdulaziz University, Jeddah. The authors, therefore, acknowledge with thanks DSR technical and financial support.

\section{References}

[1] R. P. Agarwal, Difference equations and inequalities, Theory, methods, and applications, Second edition, Monographs and Textbooks in Pure and Applied Mathematics, Marcel Dekker, Inc., New York, (2000). 1

[2] M. Aloqeili, Dynamics of a rational difference equation, Appl. Math. Comput., 176 (2006), 768-774.

[3] N. Battaloglu, C. Çinar, I. Yalcinkaya, The dynamics of the difference equation $x_{n+1}=\frac{\alpha x_{n-k}}{\beta+\gamma x_{n-(k+1)}^{p}}$, Ars Combin., 97 (2010), 281-288.

[4] E. Camouzis, M. R. S. Kulenović, G. Ladas, O. Merino, Rational systems in the plane, J. Difference Equ. Appl., 15 (2009), 303-323. 1

[5] C. Çinar, On the positive solutions of the difference equation system $x_{n+1}=\frac{1}{y_{n}}, y_{n+1}=\frac{y_{n}}{x_{n-1} y_{n-1}}$, Appl. Math. Comput., 158 (2004), 303-305.

[6] D. Clark, M. R. S. Kulenović, A coupled system of rational difference equations, Comput. Math. Appl., 43 (2002), 849-867. 1

[7] S. E. Das, M. Bayram, On a system of rational difference equations, World Appl. Sci. J., 10 (2010), $1306-1312$.

[8] Q. Din, T. F. Ibrahim, K. A. Khan, Behavior of a competitive system of second-order difference equations, Sci. World J., 2014 (2014), 9 pages. 1

[9] M. DiPippo, E. J. Janowski, M. R. S. Kulenović, Global asymptotic stability for quadratic fractional difference equation, Adv. Difference Equ., 2015 (2015), 13 pages.

[10] E. M. Elabbasy, S. M. Eleissawy, Asymptotic behavior of two dimensional rational system of difference equations, Dyn. Contin. Discrete Impuls. Syst. Ser. B Appl. Algorithms, 20 (2013), 221-235.

[11] E. M. Elabbasy, H. A. El-Metwally, E. M. Elsayed, On the solutions of a class of difference equations systems, Demonstratio Math., 41 (2008), 109-122. 1 .

[12] E. M. Elabbasy, H. A. El-Metwally, E. M. Elsayed, Global behavior of the solutions of some difference equations, Adv. Difference Equ., 2011 (2011), 16 pages.

[13] S. Elaydi, An introduction to difference equations, Third edition, Undergraduate Texts in Mathematics, Springer, New York, (2005). 1

[14] M. M. El-Dessoky, The form of solutions and periodicity for some systems of third-order rational difference equations, Math. Methods Appl. Sci., 39 (2016), 1076-1092.

[15] E. M. Elsayed, M. Mansour, M. M. El-Dessoky, Solutions of fractional systems of difference equations, Ars Combin., 110 (2013), 469-479. 1

[16] M. E. Erdogan, C. Cinar, I. Yalcinkaya, On the dynamics of the recursive sequence $x_{n+1}=$ $\frac{x_{n-1}}{\beta+\gamma x_{n-2}^{2} x_{n-4}+\gamma x_{n-2} x_{n-4}^{2}}$, Comput. Math. Appl., 61 (2011), 533-537. 
[17] M. Garić-Demirović, M. Nurkanović, Dynamics of an anti-competitive two dimensional rational system of difference equations, Sarajevo J. Math., 7 (2011), 39-56.

[18] E. A. Grove, G. Ladas, Periodicities in nonlinear difference equations, Advances in Discrete Mathematics and Applications, Chapman \& Hall/CRC, Boca Raton, FL, (2005).

[19] E. A. Grove, G. Ladas, L. C. McGrath, C. T. Teixeira, Existence and behavior of solutions of a rational system, Comm. Appl. Nonlinear Anal., 8 (2001), 1-25. 1

[20] T. F. Ibrahim, Periodicity and analytic solution of a recursive sequence with numerical examples, J. Interdiscip. Math., 12 (2009), 701-708. 1

[21] V. L. Kocić, G. Ladas, Global behavior of nonlinear difference equations of higher order with applications, Mathematics and its Applications, Kluwer Academic Publishers Group, Dordrecht, (1993).

[22] M. R. S. Kulenović, Z. Nurkanović, Global behavior of a three-dimensional linear fractional system of difference equations, J. Math. Anal. Appl., 310 (2005), 673-689. 1

[23] A. S. Kurbanli, On the behavior of solutions of the system of rational difference equations $x_{n+1}=$ $\frac{x_{n-1}}{\gamma_{n} x_{n-1}-1}, \gamma_{n+1}=\frac{\gamma_{n-1}}{x_{n} \gamma_{n-1}-1}, z_{n+1}=\frac{1}{\gamma_{n} z_{n}}$, Adv. Difference Equ., 2011 (2011), 8 pages.

[24] A. S. Kurbanli, C. Çinar, I. Yalçinkaya, On the behavior of positive solutions of the system of rational difference equations $x_{n+1}=\frac{x_{n-1}}{y_{n} x_{n-1}+1}, y_{n+1}=\frac{y_{n-1}}{x_{n} y_{n-1}+1}$, Math. Comput. Modelling, 53 (2011), 1261-1267. 1

[25] A. Y. Özban, On the system of rational difference equations $x_{n+1}=a / y_{n-3}, y_{n+1}=b y_{n-3} / x_{n-q} y_{n-q}$, Appl. Math. Comput., 188 (2007), 833-837. 1

[26] O. Özkan, A. S. Kurbanli, On a system of difference equations, Discrete Dyn. Nat. Soc., 2013 (2013), 7 pages.

[27] N. Touafek, E. M. Elsayed, On the periodicity of some systems of nonlinear difference equations, Bull. Math. Soc. Sci. Math. Roumanie (N.S.), 55 (2012), 217-224. 1

[28] C.-Y. Wang, S. Wang, W. Wang, Global asymptotic stability of equilibrium point for a family of rational difference equations, Appl. Math. Lett., 24 (2011), 714-718.

[29] I. Yalcinkaya, On the global asymptotic behavior of a system of two nonlinear difference equations, Ars Combin., 95 (2010), 151-159.

[30] Y. Yang, L. Chen, Y.-G. Shi, On solutions of a system of rational difference equations, Acta Math. Univ. Comenian. (N.S.), 80 (2011), 63-70.

[31] X. F. Yang, Y. X. Liu, S. Bai, On the system of high order rational difference equations $x_{n}=\frac{a}{y_{n-p}}, y_{n}=$ $\frac{b y_{n-p}}{x_{n-q} y_{n-q}}$, Appl. Math. Comput., 171 (2005), 853-856. 1

[32] Z. H. Yuan, L. H. Huang, All solutions of a class of discrete-time systems are eventually periodic, Appl. Math. Comput., 158 (2004), 537-546. 1

[33] Q. H. Zhang, J. Z. Liu, Z. G. Luo, Dynamical behavior of a system of third-order rational difference equation, Discrete Dyn. Nat. Soc., 2015 (2015), 6 pages. 1

[34] Y. Zhang, X. F. Yang, G. M. Megson, D. J. Evans, On the system of rational difference equations $x_{n}=A+$ $\frac{1}{y_{n-p}}, y_{n}=A+\frac{y_{n-1}}{x_{n-r} y_{n-s}}$, Appl. Math. Comput., 176 (2006), 403-408. 1 